\title{
The Gigantocellular Reticular Nucleus Plays a Significant Role in Locomotor Recovery after Incomplete Spinal Cord Injury
}

\author{
${ }^{\circledR}$ Anne K. Engmann, ${ }^{\circledR}$ Flavio Bizzozzero, Marc P. Schneider, Dario Pfyffer, Stefan Imobersteg, \\ Regula Schneider, Anna-Sophie Hofer, Martin Wieckhorst, and Martin E. Schwab \\ Department of Health Sciences and Technology, ETH Zurich, Brain Research Institute, University of Zurich, 8057 Zurich, Switzerland
}

Traditionally, the brainstem has been seen as hardwired and poorly capable of plastic adaptations following spinal cord injury (SCI). Data acquired over the past decades, however, suggest differently: following SCI in various animal models (lamprey, chick, rodents, nonhuman primates), different forms of spontaneous anatomic plasticity of reticulospinal projections, many of them originating from the gigantocellular reticular nucleus (NRG), have been observed. In line with these anatomic observations, animals and humans with incomplete SCI often show various degrees of spontaneous motor recovery of hindlimb/leg function. Here, we investigated the functional relevance of two different modes of reticulospinal fiber growth after cervical hemisection, local rewiring of axotomized projections at the lesion site versus compensatory outgrowth of spared axons, using projection-specific, adeno-associated virus-mediated chemogenetic neuronal silencing. Detailed assessment of joint movements and limb kinetics during overground locomotion in female adult rats showed that locally rewired as well as compensatory NRG fibers were responsible for different aspects of recovered forelimb and hindlimb functions (i.e., stability, strength, coordination, speed, or timing). During walking and swimming, both locally rewired as well as compensatory NRG plasticity were crucial for recovered function, while the contribution of locally rewired NRG plasticity to wading performance was limited. Our data demonstrate comprehensively that locally rewired as well as compensatory plasticity of reticulospinal axons functionally contribute to the observed spontaneous improvement of stepping performance after incomplete SCI and are at least partially causative to the observed recovery of function, which can also be observed in human patients with spinal hemisection lesions.

Key words: motor recovery; plasticity; regeneration; reticulospinal tract; spinal cord injury; sprouting

Significance Statement

Following unilateral hemisection of the spinal cord, reticulospinal projections are destroyed on the injured side, resulting in impaired locomotion. Over time, a high degree of recovery can be observed in lesioned animals, like in human hemicord patients. In the rat, recovery is accompanied by pronounced spontaneous plasticity of axotomized and spared reticulospinal axons. We demonstrate the causative relevance of locally rewired as well as compensatory reticulospinal plasticity for the recovery of locomotor functions following spinal hemisection, using chemogenetic tools to selectively silence newly formed connections in behaviorally recovered animals. Moving from a correlative to a causative understanding of the role of neuroanatomical plasticity for functional recovery is fundamental for successful translation of treatment approaches from experimental studies to the clinics.

Received Feb. 26, 2020; revised Sep. 14, 2020; accepted Sep. 18, 2020.

Author contributions: A.K.E., A.-S.H., and M.E.S. designed research; A.K.E., F.B., M.P.S., D.P., S.I., and R.S. performed research; M.W. contributed unpublished reagents/analytic tools; A.K.E., F.B., D.P., and A.S.H. analyzed data; A.K.E. and M.E.S. wrote the paper.

This study was supported by grants from the Swiss National Science Foundation (Grant 3100A0-12225272), the European Research Council (Advanced Grant 294115 NOGORISE), and the Christopher and Dana Reeve Foundation (Spinal Cord Consortium). We thank the viral vector facility of the University of North Carolina and Prof. Olivier Raineteau for supplying us with viral vectors. We also thank the Centre for Microscopy and Image Analysis of the University Zurich for access to and support with their microscopes. In addition, we thank Anna Jeske and Nicole Rappo for their technical support on this project, as well as Oliver Weinmann and Priya Veeraraghavan for fruitful scientific discussions.
The authors declare no competing financial interests.

A.K. Engmann's present address: Department of Stem Cell and Regenerative Biology at Harvard University, Cambridge, MA 02138

M.E. Schwab's present address: The Institute for Regenerative Medicine, University of Zurich, 8952 Schlieren, Switzerland.

Correspondence should be addressed to Anne K. Engmann at anne_engmann@harvard.edu or Martin E. Schwab at schwab@irem.uzh.ch.

https://doi.org/10.1523/JNEUROSCI.0474-20.2020

Copyright $\odot 2020$ the authors 


\section{Introduction}

Crude motor functions, like overground locomotion and posture, are generated by an intricate interplay of various midbrain, brainstem, and spinal circuits: key structures in the midbrain [subthalamic (Sinnamon and Stopford, 1987) and mesencephalic locomotor region (Shik et al., 1966; Skinner and Garcia-Rill, 1984; Bachmann et al., 2013; Roseberry et al., 2016; Caggiano et al., 2018)] as well as the cerebellum (Mori et al., 1998) have been shown to be crucial for the initiation and maintenance of coordinated limb movements via a relay of neuronal output to the reticular formation (Orlovskii, 1970; Steeves et al., 1987; Noga et al., 1988, 2003; Jordan et al., 2008). The reticular formation is an assortment of various motor-related nuclei in the pons and medulla oblongata, among which the gigantocellular reticular nucleus (NRG) has been characterized as a main output center (Brownstone and Chopek, 2018). Descending projections originating from the NRG join the reticulospinal tract (ReST), which runs in the ventrolateral funiculus of the spinal cord and innervates spinal laminae VII, VIII, and, to a limited extent, IX and X. The ReST relays motor-relevant neuronal commands from the reticular formation to central pattern generator units and the motor neuron columns in the spinal cord (Liang et al., 2016).

Following incomplete spinal cord injury (SCI), experimental studies in various animal models (Raineteau and Schwab, 2001; Fouad and Tse, 2008) as well as clinical observations in human patients (Dobkin et al., 2007; Fawcett et al., 2007) have shown surprising levels of spontaneous recovery of crude motor functions, even with very limited numbers of spared fibers. In animal models, these behavioral findings have been correlated with anatomic observations: upon incomplete SCI in lamprey, chicken, rodents, and nonhuman primates, bulbospinal and intraspinal circuits show pronounced spontaneous plasticity (Lurie and Selzer, 1991; Hasan et al., 1993; Ballermann and Fouad, 2006; Pettersson et al., 2007; Vavrek et al., 2007; Courtine et al., 2008; Zaaimi et al., 2012; Baker and Perez, 2017; May et al., 2017; Asboth et al., 2018). In general, two different modes of neuronal outgrowth can be distinguished following incomplete SCI: local rewiring of lesioned axons and compensatory fiber growth from spared projections. Following incomplete spinal injury in rodents, bulbospinal projections are able to spontaneously generate both types of plasticity (Ballermann and Fouad, 2006; Courtine et al., 2008; May et al., 2017; Asboth et al., 2018). Previously, we have reported that, following a $\mathrm{C} 4 / \mathrm{C} 5$ unilateral hemisection lesion in rats, these two types of spontaneous neuroanatomical adaptations can originate in parallel from the same nucleus in the brainstem, the NRG: midline-crossing NRG fibers from the intact side in the cervical as well as lumbar enlargement (compensatory NRG sprouting; Zörner et al., 2014) and locally rewiring plasticity from injured NRG axons that form local relay connections to propriospinal neurons, which themselves showed pronounced spontaneous plasticity in response to axotomy, thereby forming a detour pathway around the lesion site (Filli et al., 2014). Recent neuromodulative approaches, using light (Fenno et al., 2011) or pharmacological compounds (Gompf et al., 2015; Roth, 2016) to specifically manipulate neuronal activity of a defined subset of neurons, now allow causal testing of the contribution of defined forms of anatomic rewiring to the observed functional recovery in the injured spinal cord.

Here, we investigate the functional relevance of locally rewired versus compensatory NRG plasticity for the recovery of overground locomotion observed after spinal hemisection using projection-specific intersectional viral genetics to introduce the silencing hM4Di receptor into either axotomized or spared NRG neurons. Detailed kinematic assessment of the locomotor performance of the animals following SCI in the presence and absence of designer receptors exclusively activated by designer drugs (DREAAD)-induced neuronal silencing showed that compensatory as well as locally rewired reticulospinal plasticity causally contribute to the observed functional recovery of walking, wading, and swimming.

\section{Materials and Methods}

Animals, housing, and preparation for behavioral testing. All animal experiments were performed in accordance with the rules and guidelines and with approval of the Veterinary Office of Zurich in Switzerland. Adult, female Lewis rats (weight, 230-250 g; Janvier; RRID:RGD_ 737932) were housed under standard conditions in groups of four, with a $12 \mathrm{~h}$ dark/light cycle and ad libitum access to food and water. Based on the principles of $3 \mathrm{R}$, this study was limited to female rats. In male animals, bladder management after spinal cord injury is very challenging because of their longer urinary tract and the prostate, resulting in a high rate of severe and treatment-resistant bladder complications.

Before starting experiments, animals were tamed and trained in the overground locomotor tasks walking, wading, and swimming using the setup outlined in Figure 1D. To enable detailed kinematic tracking, prominent anatomic structures were marked as described previously (Zörner et al., 2010). Briefly, under isoflurane anesthesia (2-3\% in air; Attane, Piramol Healthcare), the fur overlying prominent bone structures of the forelimb and hindlimb was shaved and the skin disinfected using $70 \%$ ethanol. The following bone structures were permanently marked on both body sides of each animal using small round tattoos (Hugo Sachs Electronik/Harvard Apparatus): vertebral border of the scapula (shoulder blade); tip of the humerus (shoulder joint); iliac crest; and greater trochanter (hip). To enhance the visibility of the landmarks, the tattoos were highlighted using a black permanent marker pen before each behavioral testing session. During this preparation of the animal, the following additional anatomic landmarks were also labeled bilaterally using a permanent marker pen: wrist; fifth metacarpal head $(\mathrm{MCH})$ of the hand; tip of the fourth finger; lateral malleolus (ankle); metatarsophalangeal joint of fifth toe (MTP); tip of the fourth toe; tail base; tip of the tail as well as one-third and two-thirds of the tail length (Extended Data Fig. 1-1B).

Postoperative care. After any surgical intervention animals were closely monitored daily, weight was documented, and additional food and water within the home cage was supplied if necessary. After SCI, manual bladder management was performed twice daily, until animals recovered independent voiding. Animals received daily injections of antibiotics ( $5 \mathrm{mg} / \mathrm{kg}$ body weight; Baytril, Bayer) and analgesics $(2.5 \mathrm{mg} /$ $\mathrm{kg}$ body weight; Rimadyl, Pfizer) for at least $7 \mathrm{~d}$ after SCI. Dehydration was treated with subcutaneous injections of prewarmed glucosaline solution (B Braun). After stereotaxic injections, animals received daily subcutaneous injections of antibiotics ( $5 \mathrm{mg} / \mathrm{kg}$ body weight; Baytril, Bayer) and analgesics ( $2.5 \mathrm{mg} / \mathrm{kg}$ body weight; Rimadyl, Pfizer) for at least $3 \mathrm{~d}$.

Spinal cord injury. Animals were deeply anesthetized using intramuscular application of a combination of medetomidin $(0.105 \mathrm{mg} / \mathrm{kg}$ body weight; Domitor, Provet), midazolam $(1.4 \mathrm{mg} / \mathrm{kg}$ body weight; Dormicum, Roche), and fentanyl ( $0.007 \mathrm{mg} / \mathrm{kg}$ body weight; Kantonsapotheke Zürich). They additionally received $1 \mathrm{mg} / \mathrm{kg}$ Fortecortin (Merck Serono) intramuscularly to optimize the analgesic coverage and to prevent swelling of CNS tissue after injury. The skin overlying the cervical spinal cord was shaved, disinfected, and opened with a scalpel blade. The muscle layers covering the vertebral column were blunt dissected, and the vertebrae $\mathrm{C} 4$ and $\mathrm{C} 5$ were cleaned of remaining connective tissue. The ligament between vertebrae $\mathrm{C} 4$ and C5 was opened, granting access to the spinal cord. After locally opening the dura, a unilateral, right-sided hemisection was performed using a $15^{\circ}$ scalpel (catalog \#P-715, MicroFEATHER Safety Razor). Bleeding was stopped using Gelfoam (Pfizer), and the corresponding muscle layers were closed using silk thread (Silkam, B Braun). After disinfection of the wound with BETADINE (Mundipharma), the skin was sutured using silk thread (Silkam, B Braun). Animals were allowed to recover on a heat blanket for 30-60 min before the anesthesia was antagonized using subcutaneous 
A

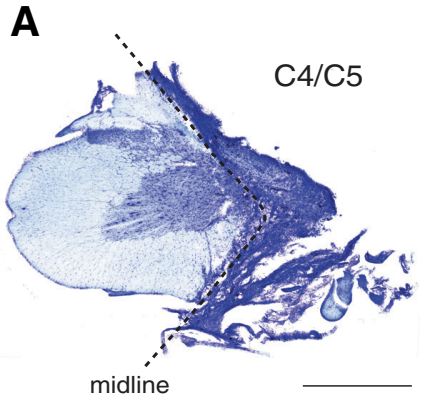

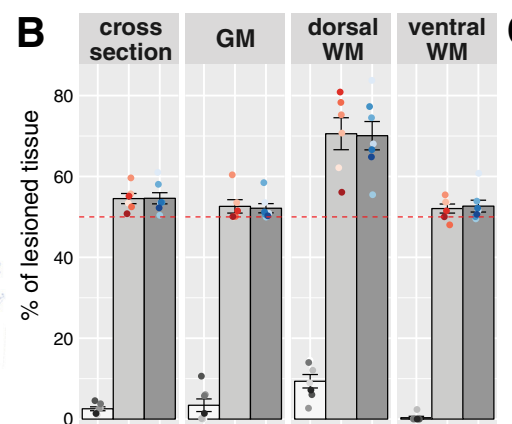
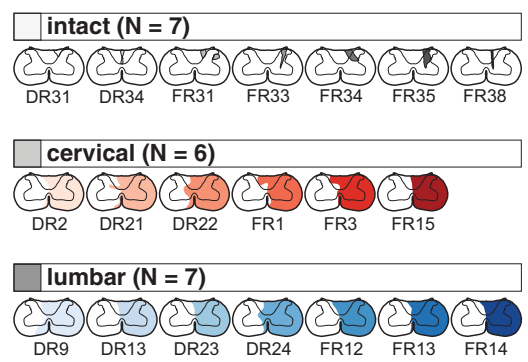

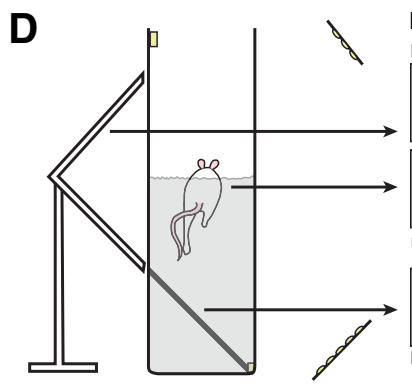

high speed video:

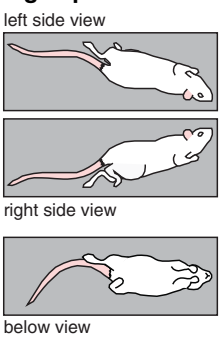

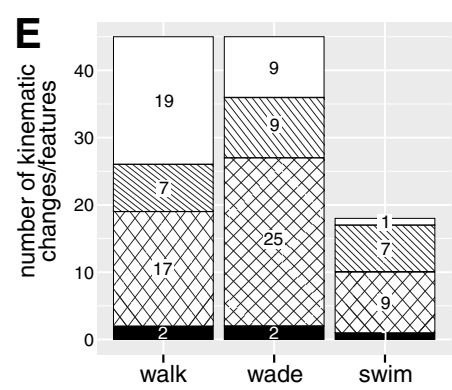
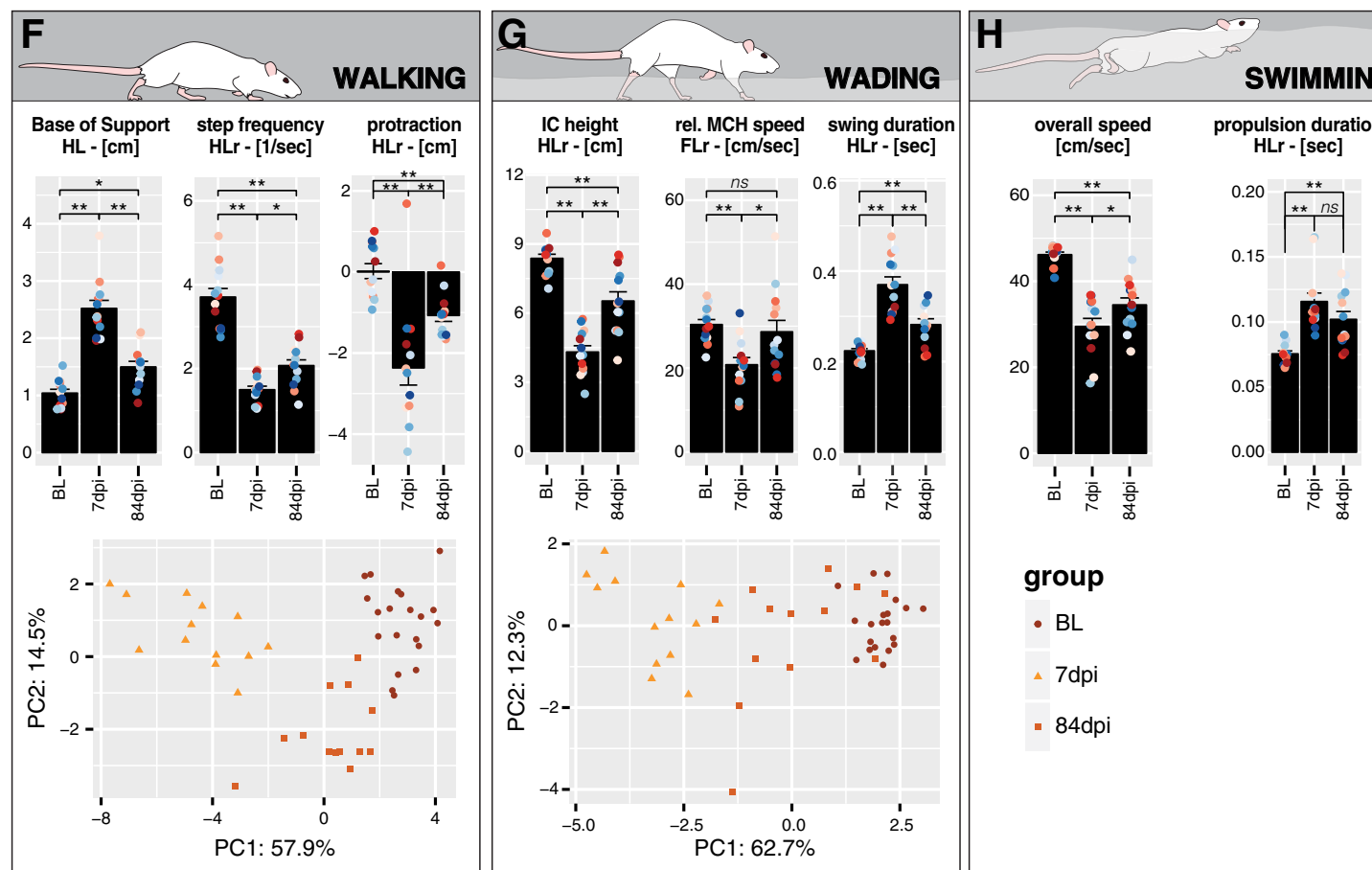

group

- BL

$\triangle 7 \mathrm{dpi}$

- 84dpi

RecoveryProfile

A $\square$ lesion-induced impairment,

II followed by recovery

31 no impairment

impaired, no recovery

others

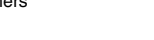

Figure 1. Unilateral hemisection lesion at spinal level $C 4 / 5$ in adult rats leads to severe acute impairment of walking, wading, and swimming followed by pronounced spontaneous recovery of a distinct subset of kinematic features. $A$, Cross section of the spinal cord at level $C 4 / 5$, stained with cresyl violet (Nissl), shows the unilateral, right-sided hemisection 18 weeks postinjury. The completely destroyed right hemicord is replaced by a fibrotic scar. Scale bar, $1 \mathrm{~mm}$. B, Quantification of tissue damage of all animals enrolled in this study (mean \pm SEM) for the complete spinal cross section (cross section), bilateral gray matter (GM), dorsal white matter (WM), and ventral WM. The red line indicates the optimal lesion extent of a hemisection (50\%). Color codes of groups and single animals are defined in Figure 1C. C, Maximal spinal lesion extent for all animals of this study. Each animal has a unique color identifier, which is consistent with any other graph showing single animal data. Minor damage $(<20 \%)$ of dorsal WM occasionally resulted from laminectomy or viral injections also in the intact control group. $\boldsymbol{D}$, Behavioral setup to assess walking, wading, and swimming performance. The animal moves in a glass tank while being recorded with a high-speed camera. Mirrors enable parallel recording of the right-side, leftside, and below view of movement in the same video file. Distinct marked limb joints are tracked (Extended Data Figure 1-1B), allowing the calculation of an array of kinematic parameters, subsequently called features, reflecting the locomotor performance of the animal (Zörner et al., 2010). For a full list of assessed features please refer to Extended Data Figure 1-1, A and C. $\boldsymbol{E}$, Classification of the different kinematic changes/features observed for walking, wading, and swimming, based on the pattern of lesion-induced impairment and recovery over time (recovery profile). This classification was performed on the basis of the statistical testing results (one-way ANOVA, Tukey's post hoc test) comparing locomotor performance at BL, acutely (7 dpi) and chronically (84 dpi) after injury. Features that show a significant initial impairment and subsequent recovery over time are considered to be class $\mathrm{A}$ features. Features that do not show significant lesion-induced changes are considered class $B$, and impairments without recovery are considered class C features. A comprehensive visualization of class $A$ features is shown in Figures $3 G$ and $4 H$. $\boldsymbol{F}$, Representative parameters for the locomotor modality walking (mean \pm SEM, one-way ANOVA followed by Tukey post hoc testing: $n s$, not significant, ${ }^{*} p<0.05,{ }^{* *} p<0.001$; $N=13$ ). The three graphs in the top panel show quantifications of the base of support of the hindlimbs (HLs), the step frequency, and the protraction of the right hindlimb (HLr) over time. Compared with BL performance, all three shown features are significantly impaired at the acute time point ( $7 \mathrm{dpi})$ and have significantly recovered at chronic stages after injury $(84 \mathrm{dpi})$, and are therefore representative class A features. The bottom panel shows the results of a PCA applied to all class A features of the walking dataset. The first principal component (PC1) can explain 
application of an antidote $(0.75 \mathrm{mg} / \mathrm{kg}$ body weight; Antisedan, Provet; and $1 \mathrm{mg} / \mathrm{kg}$ body weight; Anexate, Roche).

Stereotaxic injections of adeno-associated virus in the brainstem. Animals were anesthetized using intramuscular application of a combination of medetomidin $(0.15 \mathrm{mg} / \mathrm{kg}$ body weight; Domitor, Provet), midazolam $(2 \mathrm{mg} / \mathrm{kg}$ body weight; Dormicum, Roche) and fentanyl $(0.005 \mathrm{mg} / \mathrm{kg}$ body weight; Kantonsapotheke Zürich), and were head fixed in a stereotaxic frame. The skin overlying the skull was shaved, disinfected, and opened. Connective tissue on the skull was removed and the head was leveled, ensuring $\lambda$ and bregma to be in one mediolateral and dorsoventral plane. After performing a craniectomy, adeno-associated virus (AAV) encoding a Cre-dependent version of the inhibitory DREADD receptor hM4Di [titer, $4 * 10^{12}$ infectious particles $/ \mathrm{ml}$; AAV2.1-EF1a::DIO-hM4Di-mCherry, UNC Vector Core (University of North Carolina, Chapel Hill, NC)] was injected into the NRG using 33 ga needles (NanoFil, World Precision Instruments) attached to a microinjection pump (model UMC4, World Precision Instruments) at the following coordinates: anteroposterior, $-4.6 \pm 0.8 \mathrm{~mm}$; mediolateral (ML), $0.9 \mathrm{~mm}$ from $\lambda$; and dorsoventral (DV), $-8.0 \mathrm{~mm}$ from the dura. Ipsilesional/contralesional positioning of the injection site was adapted according to the group assignment of the animal (Fig. $2 A, B$ ). At each injection site, two repeated injections of $50 \mathrm{nl}$ each were performed at the same position, with a 5 min pause between repeated injections. The needle was left in place for 5 min following the second injection and before retracing the needle to prevent backflow of the virus. After retracting the needle, the wound was disinfected using BETADINE (Mundipharma), and the skin was closed using wound clips (World Precision Instruments). Animals were allowed to recover on a heat blanket for 30-60 min before anesthesia was antagonized using subcutaneous application of antidote $(0.75 \mathrm{mg} / \mathrm{kg}$ body weight; Antisedan, Provet; and $1 \mathrm{mg} / \mathrm{kg}$ body weight; Anexate, Roche).

Stereotaxic injections of $A A V$ in the spinal cord. Animals were anesthetized using intramuscular application of a combination of medetomidin $(0.15 \mathrm{mg} / \mathrm{kg}$ body weight; Domitor, Provet), midazolam $(2 \mathrm{mg} / \mathrm{kg}$ body weight; Dormicum, Roche) and fentanyl $(0.005 \mathrm{mg} / \mathrm{kg}$ body weight; Kantonsapotheke Zürich). The skin overlying the cervical and/or lumbar spinal cord was shaved, disinfected, and opened. The muscle layers covering the vertebral column were opened using blunt dissection. A laminectomy of the vertebrae C3 and C4 and/or T12 and T13 (corresponding to spinal levels L1 to L4) was performed. The animal was positioned in the stereotaxic frame and eight injections of $100 \mathrm{nl}$ each of the virus AAV2.6-Pgk::Cre (generous gift from Prof. Olivier Raineteau, Stem Cell and Brain Research Institute, Lyon, France) were performed in the cervical and/or lumbar spinal cord (Fig. $2 A, B$ ) using a 35 ga needle (NanoFil, World Precision Instruments) attached to a microinjection system (UMC4, World Precision Instruments). The following coordinates were targeted in the cervical spinal cord, as follows: ML, $0.9 \mathrm{~mm}$ from the midline; $\mathrm{DV},-1.6 \mathrm{~mm}$ from the spinal surface. In the lumbar spinal cord injections were made at ML $0.6 \mathrm{~mm}$ from the midline and DV $-1.2 \mathrm{~mm}$ from the spinal surface. Single injection sites were spaced by $500 \mu \mathrm{m}$. After injections were completed, the corresponding muscle

$57.9 \%$ of the variance in the dataset, and the color grouping shows that this component robustly separates the three different time points (BL, 7 and 84 dpi). $\mathbf{G}$, Representative class A movement parameters for the locomotor modality wading (mean \pm SEM; one-way ANOVA followed by Tukey post hoc testing: $n s$, not significant, ${ }^{*} p<0.05$, ${ }^{* *} p<0.001$, $N=13$ ). The three graphs in the top panel show quantifications of the height of the iliac crest (IC) of the HLr, the relative speed of the fifth metacarpal head (MCH), and the swing phase duration of the HLr (ipsilesional) over time. All three features show significant lesion-induced impairment ( 7 dpi) and subsequent partial or full recovery (84 dpi). The bottom panel shows the results of a PCA applied to all class A features of the wading dataset. The PC1 can explain $62.7 \%$ of the variance in the dataset, and the color grouping shows that this component robustly separated the three different time points (BL, 7 and $84 \mathrm{dpi})$. $\boldsymbol{H}$, The one class A parameter for swimming (mean \pm SEM, one-way ANOVA followed by Tukey post hoc testing: $n s$, not significant, ${ }^{*} p<0.05$, $\left.{ }^{* *} p<0.001 ; N=13\right)$. The two graphs in the top panel show quantifications of the overall speed of the animals (class A feature) and the propulsion phase duration of the HLr (ipsilesional) over time, as an example of a class ( feature. rel., Relative. layers were closed using silk thread (Silkam, B Braun), the wound was disinfected, and the skin was sutured using silk thread (Silkam, B Braun). Animals were allowed to recover on a heat blanket for 30 $60 \mathrm{~min}$ before the anesthesia was antagonized using subcutaneous application of antidote $(0.75 \mathrm{mg} / \mathrm{kg}$ body weight; Antisedan, Provet; and $1 \mathrm{mg} / \mathrm{kg}$ body weight; Anexate, Roche).

Behavioral testing of overground locomotor function (with/without clozapine $\mathrm{N}$-oxide treatment). After preparing the animal for behavioral assessment, at least three high-quality videos per animal were recorded as described previously (Zörner et al., 2010). Briefly, animals were placed in a tank made of duplex nonreflecting glass $(150 \mathrm{~cm}$ length) with one mirror positioned at a $45^{\circ}$ angle at the bottom and two mirrors positioned at $90^{\circ}$ angles behind the tank (Fig. $1 D$, scheme). Illumination was ensured using an LED panel positioned in front of the setup and two additional LED strips, one located at the bottom and one attached to the top inside the tank. For the assessment of walking or wading performance, a runway also made of duplex nonreflecting glass (120 cm length) was positioned in the tank. For wading, the tank was filled with water $\left(20-22^{\circ} \mathrm{C}\right)$ to reach a level of $5 \mathrm{~cm}$ above the runway. For assessment of swimming performance, the runway was removed, and the water level was increased to reach $24 \mathrm{~cm}$ over the bottom of the tank. Videos were recorded using a high-speed camera at $200 \mathrm{~Hz}$ (A504kc Color Camera, Basler) with an exposure time of $1 \mathrm{~ms}$ and an aperture opening of 5.6. High-quality videos had to fulfil the following requirements: the animal had to walk/wade/swim in a straight line at constant speed performing at least three to four complete step cycles or five to six complete swim cycles within the field of view with all limbs and feet visible at any point during the video. For behavioral testing under the influence of the DREADD ligand clozapine $\mathrm{N}$-oxide (CNO) or vehicle (Veh), animals were injected intraperitoneally with $10 \mathrm{mg} / \mathrm{kg} \mathrm{CNO}$ (dissolved in $0.9 \%$ $\mathrm{NaCl}$ solution; Enzo Life Sciences) or the corresponding volume of the vehicle solution $(0.6 \%$ methanol in $0.9 \% \mathrm{NaCl}$ solution) $30 \mathrm{~min}$ before kinematic testing. All recordings for walking, wading, and swimming of one animal were performed within a time window of $45 \mathrm{~min}$ to ensure comparable levels of CNO.

Video analysis and calculation of kinematic parameters. For each animal, one behavioral video representing the best possible locomotor performance was selected and debayered using a custom-made LabView script (LabView version 8.6/2008). Kinematic tracking data were generated using manual tracking in Fiji [Image), version 1.50g (64-bit version)]. Briefly, the frames of onset (the first complete paw contact with the runway for walking and wading, minimal angle between knee-ankleMTP for swimming) and offset (MTP/MCH liftoff for walking and wading, maximal limb extension for swimming) of each limb were determined. The time point of midstance was calculated as the mathematical mean between onset and offset.

For walking and wading, at the onset and offset of each step cycle for each paw, the position of the mouth and anus, thenar and $\mathrm{MCH}$ (forelimb), or heel and MTP (hindlimb) was tracked in the below view of the video. In the same frames, the position of the MCH or MTP and shoulder blade or iliac crest (for forelimb and hindlimb, respectively) was determined in the front and back side views using Fiji. Additionally, at the time point of midstance, the shoulder blade and the runway surface were tracked. The acquired values for $x$ - and $y$-coordinates of the tracked points were exported from Fiji, and a range of locomotor parameters was calculated using MATLAB version R2015b for mathematical definitions (Extended Data Fig. 1-1A).

For swimming, at the time points of hindlimb onset and offset, mouth and anus were tracked in the below view. In the same frames, the coordinates of nose tip and tail base, as well as the location of iliac crest, hip, ankle, and MTP were determined in the front and back side views using Fiji. Data were exported for calculation of the parameters shown in Extended Data Fig. 1-1C using MATLAB version R2015b. All calculated values in pixels were normalized to distances in centimeters. The acquired data were statistically analyzed and plotted in R studio (version 1.1.456; R Studio)

Perfusion and tissue processing. Animals were transcardially perfused using Ringer's solution supplemented with $1 \%$ heparin (B Braun) followed by $4 \%$ paraformaldehyde (PFA) solution in $0.1 \mathrm{M} \mathrm{PB}$ containing 
A

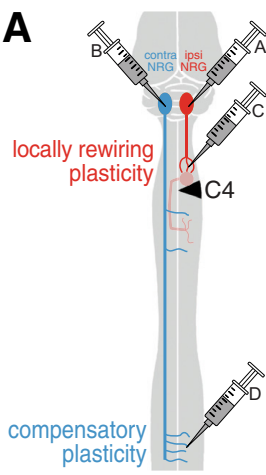

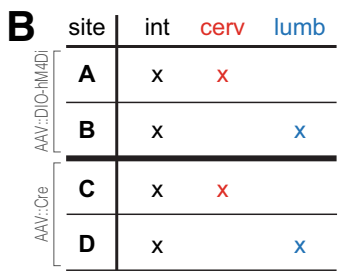

C AAV2.6[Pgk::Cre]
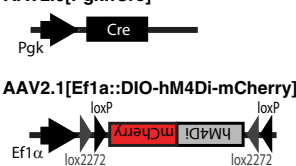

D

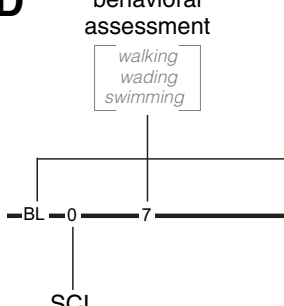

$\mathrm{SCl}$

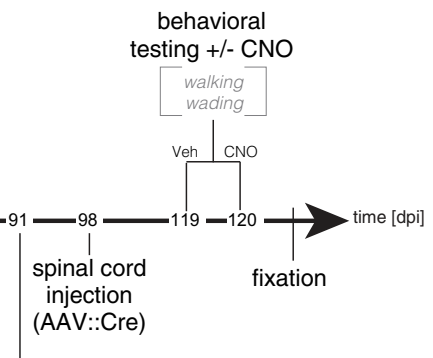

brainstem

injection

(AAV::DIO-hM4Di)

\section{E}

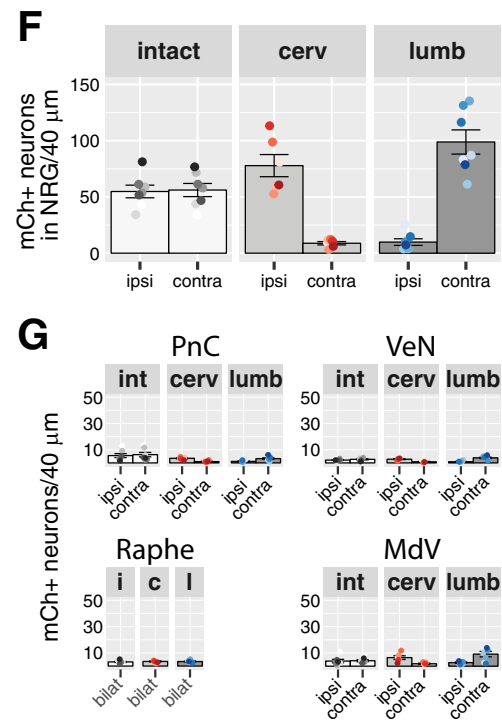

Figure 2. Intersectional viral genetics allow projection-specific introduction of DREADD constructs into NGR neurons with locally rewiring or compensatory spinal fiber growth, respectively, in chronically recovered rats with cervical hemisections. $\boldsymbol{A}, \boldsymbol{B}$, Two forms of spontaneous NRG spinal axonal sprouting can be detected following cervical hemisection injury: locally rewiring outgrowth from axotomized versus compensatory growth from spared NRG projections. AAV::Cre (injection sites A and B, respectively) and AAV::DI0-hM4Di virus (injection sites C and D, respectively) were injected separately to target two different types of new NRG connections (cerv and lumb) for expression of the inhibitory DREADD channel hM4Di in a projection-specific manner. $B$, Table highlighting the location of the AAV::DI0-hM4Di injection sites in the brainstem $(A, B)$ and the AAV::Cre injections in the spinal cord $(C$, D) for the separate groups. Groups cerv and lumb are spinal cord injured, group int consists of uninjured rats. C, Viral vector constructs AAV2.6[Pgk::Cre] (AAV::Cre) and AAV2.1[Ef1a::DI0-hM4Di-mCherry] (AAV::DI0-hM4Di) used for projection-specific chemogenetic priming. D, Timeline illustrating study design. Assessment of walking, wading, and swimming performance was done before $S C l$ to obtain a BL, as well as acutely ( $7 \mathrm{dpi}$ ) and chronically ( $84 \mathrm{dpi)}$ following lesioning. AAV vectors were then injected into specific sites in the brainstem and spinal cord defining the different experimental groups $(\boldsymbol{A}, \boldsymbol{B})$. Three weeks after viral injections, animals were tested for their performance in walking, wading, and swimming in the presence (CNO) and in the absence (Veh) of the hM4Di ligand CNO. $\boldsymbol{E}$, Cross section of the lower brainstem showing mCherry-positive neurons expressing both viruses located bilaterally in the NRG from an animal of the int group. Inset, High-magnification confocal image of mCherry-positive NRG neurons. Scale bars: overview, $1 \mathrm{~mm}$; inset, $100 \mu \mathrm{m}$. $\boldsymbol{F}$, Number of mCherry-expressing neurons/40 $\mu \mathrm{m}$ section in the ipsilesional (ipsi) and contralesional (contra) NRGs for the individual groups (mean \pm SEM). Higher numbers in the lesioned groups reflect higher virus uptake in the spinal cord because of sprouting. $\mathbf{G}$, Number of mCherry-expressing neurons $/ 40 \mu \mathrm{m}$ section in the ipsi and contra, or bilateral (bilat) $\mathrm{PnC}, \mathrm{VeN}$, raphe, and MdV for the individual groups (mean \pm SEM).

$5 \%$ sucrose. After postfixation overnight in 4\% PFA, the tissue was cryoprotected using $30 \%$ sucrose in $0.1 \mathrm{M} \mathrm{PB}$ for $4-5 \mathrm{~d}$. Following embedding in O.C.T. (TissueTek), coronal sections of brain and spinal cord tissue $(40 \mu \mathrm{m})$ were collected either directly on SuperFrost glass slides (Gerhard Menzel) or free floating in $0.1 \mathrm{M} \mathrm{PB}$. Free-floating sections were stored in antifreeze solution (300 g of glucose, $1000 \mathrm{ml} 0.05 \mathrm{M} \mathrm{PB}$, $600 \mathrm{ml}$ ethylene glycol) at $-20^{\circ} \mathrm{C}$ until needed.

Histologic Nissl staining and assessment of spinal lesion completeness. Serial coronal sections of the spinal cord collected on SuperFrost glass slides were warmed to $50-60^{\circ} \mathrm{C}$ on a heat plate for $5-10 \mathrm{~min}$ to ensure adhesion of tissue to the slide during the subsequent staining procedure. The sections were then rinsed in tap water and immersed in cresyl violet solution [1 g Cresyl Violet Acetate (Sigma-Aldrich) in $200 \mathrm{ml}$ doubledistilled $\mathrm{H}_{2} \mathrm{O}$ ] for 2-10 min. Afterward, they were differentiated in $70 \%$ EtOH and dehydrated (80\%, 90\%, 95\%, 100\% EtOH), followed by immersion in Xylol and coverslipping with Eukitt (O. Kindler). After drying, bright-field images were acquired using the Axio Scan.Z1 slide scanner (10× objective; Zeiss) at the Center for Microscopy and Image
Analysis at the University Zurich. Collapsed 2D reconstruction of the maximum spinal lesion extent were generated using a template (generated from intact tissue) in Fiji [ImageJ, version 1.50g (64 bit version)] and Adobe Illustrator CS6, version 16.0.3. Lesion volume, positioning, and completeness were assessed using Image and the acquired data were plotted in R studio (version 1.1.456, R Studio).

Immunohistochemical enhancement of mCherry and quantification of viral transfection efficacy in the NRG. Free-floating sections spanning the anterior-posterior extent of the NRG were washed once in $0.1 \mathrm{M} \mathrm{PB}$ and blocked for $30 \mathrm{~min}$ at RT in a 2:1 mixture of TNB blocking buffer (Müllner et al., 2008) and TBS/0.3\% Tx (block/perm solution). The anterior-posterior extent of the NRG was defined by the first section with the facial nerve fully connected in the coronal plane (anterior start of the $\mathrm{NRG}$ ) and the end of the inferior olive (posterior end of the NRG). The sections were then incubated for $48 \mathrm{~h}$ at $4^{\circ} \mathrm{C}$ with the primary antibody rabbit-anti-mCherry (1:1000 in block/perm solution; catalog \#ab167453, Abcam; RRID:AB_2571870). After one washing step in PBS for $10 \mathrm{~min}$, the sections were washed twice in $0.1 \mathrm{M} \mathrm{PB}$ for $15 \mathrm{~min}$ each and 
incubated with the biotinylated secondary antibody goat-anti-rabbit (1:300 in block/perm solution; catalog \#111-065-003, Jackson ImmunoResearch; RRID:AB_2337959) for $1-2 \mathrm{~h}$ at room temperature (RT). Afterward, the sections were again washed once for $10 \mathrm{~min}$ in PBS and two times in $0.1 \mathrm{M} \mathrm{PB}$, for $15 \mathrm{~min}$ each, and incubated for $1 \mathrm{~h}$ at RT with Streptavidin-Cy3 (1:1000 in block/perm solution; catalog \#016-160-084, Jackson ImmunoResearch; RRID:AB_2337244) and DAPI (1:10,000; catalog \#D3571, Thermo Fisher Scientific; RRID:AB_2307445). After a final washing sequence, $10 \mathrm{~min}$ in $\mathrm{PBS}$ followed by two times for $15 \mathrm{~min}$ in $0.1 \mathrm{M}$ PB, sections were immersed in $0.05 \mathrm{M}$ Tris and mounted on glass slides (Gerhard Menzel Glasbearbeitungswerk). Once the sections had dried, they were coverslipped with Mowiol supplemented with $0.25 \%$ DABCO [10\% Mowiol (Calbiochem) in $100 \mathrm{~mm}$ Tris pH 8.5, 25\% glycerol (w/v) and $0.25 \%$ DABCO] and imaged using the Axio Scan.Z1 slide scanner $(10 \times$ objective, Zeiss) at the center for microscopy of the University Zurich.

The acquired images were adjusted in brightness and contrast for optimal signal-to-noise ratio using the ZEN software (ZEN 2012, version 1.1.2.0, Carl Zeiss Microscopy) and the number of mCherry-expressing (mCherry-positive) cells was counted on the ipsilateral and contralateral side of the NRG, caudal pons (PnC), medullary reticular nucleus ventral part $(\mathrm{MdV})$, and medial and lateral vestibular nuclei $(\mathrm{VeN})$, and bilaterally for the raphe. The specific nuclei were defined using prominent landmarks based on the Paxinos and Watson (1998) rat brain atlas. Results were plotted as bar graphs representing the group mean \pm SEM using R Studio (version 1.1.456; R Studio).

Experimental design and statistical analysis. This study was performed with a total of 20 adult female Lewis rats, which were assigned to an intact control group $(N=7)$ and two different lesioned groups (cervical, $N=6$; lumbar, $N=7$ ), based on their initial behavioral impairment [7 d postinjury (dpi)] and subsequent recovery ( $84 \mathrm{dpi}$ ) to ensure comparability of the different groups. The general experimental design was as follows (Fig. 2D): animals were acclimatized, tamed, and trained in the behavioral testing setup before the start of the study. Behavioral assessment in the locomotor modalities walking, wading, and swimming was performed before the SCI to obtain a baseline (BL), as well as acutely ( $7 \mathrm{dpi}$ ) and chronically ( $84 \mathrm{dpi}$ ) following lesion. AAV vectors were then injected into specific sites in the brainstem and spinal cord, thereby defining the different testing groups (Fig. $2 A, B$ ). Three weeks after these surgeries, animals were tested for their performance in walking, wading, and swimming in the presence $(\mathrm{CNO})$ and absence (Veh) of the hM4Di ligand CNO. Finally, animals were transcardially perfused and the CNS tissue was collected for histology. Along the course of the experiment, the following exclusion criteria were applied: animals with too small lesions ( $>2.5 \%$ ventral white matter sparing) or too big lesions $(>65 \%$ of total gray matter, or $>20 \%$ contralesional dorsal white matter damage resulting from the SCI or from the virus injections) were excluded from further analysis (a total of three lesioned and four intact animals were removed from the study at this point), as well as animals with insufficient viral transduction of the NRG or with spinal injection sites not being strictly unilateral (as assessed by positioning of the needle tract; no animals had to be excluded here).

All graphs and statistical analyses were generated using R Studio (version 1.1.456, R Studio). To assess the statistical significance of initial impairment and the subsequent recovery of behavioral performance, a one-way repeated-measures ANOVA with Tukey's post hoc test for multiple comparison was performed $\left({ }^{*} p<0.05,{ }^{*} p<0.001\right)$. Based on these statistical results, the different behavioral features were categorized into "class A" (lesion-induced impairment, followed by recovery), "class B" (no impairment), and "class C" (impairment, no recovery). As the focus of the study was on behaviors that recovered spontaneously after the injury, only class A features were used for the subsequent analysis. The power of the filtered feature dataset to discriminate between the different time points was assessed using principal component analysis. The statistical significance of behavioral performance post-viral injection of lesioned and recovered animals with and without $\mathrm{CNO}$ was tested using a paired, two-sided Student's $t$ test $\left({ }^{*} p<0.05,{ }^{* *} p<0.001\right)$. The statistical significance of behavioral performance comparing the pre- and postviral injection of intact rats with and without $\mathrm{CNO}$ was tested using a one-way repeated-measures ANOVA with Tukey's post hoc testing. "Effect size" was defined as a metric to generate direct visualization for changes in behavioral performance at acute and chronic stages, as well as in the presence of vehicle or CNO: the effect size of the SCI-induced impairment and spontaneous recovery was calculated for each feature as the difference between behavioral performance at 84 and $7 \mathrm{dpi}$ (pre), whereas the effect size of the CNO-mediated changes was defined as the difference in behavioral under vehicle and CNO treatment (post). Fingerprint graphs were plotted using the mean of the effect size normalized to a $[-1,1]$ interval, with the whiskers representing the $95 \%$ confidence interval calculated using the $t$ values, corrected for the $N$ number of the respective group. Correlations in scatterplots are based on a Pearson's linear model.

\section{Results}

A right-sided, unilateral spinal cord hemisection was performed at cervical level C4/C5 in adult, female Lewis rats (Fig. 1A). The spinal lesion site was analyzed for completeness and animals with incomplete hemisection lesions $(>2.5 \%$ ventral white matter sparring) or pronounced amounts of secondary damage ( $>65 \%$ total gray matter or intact side dorsal column damage) were excluded from the study (Fig. 1B,C). A total of three animals was excluded because of lesion size, while 13 injured animals were included for further analysis. During the course of the experiment, animals of the intact control group received intraspinal injections in the cervical as well as the lumbar enlargement, which in some cases resulted in minor damage of the dorsal funiculus. Intact animals with $>20 \%$ dorsal white matter damage were excluded from further analysis (a total of four intact animals was excluded based on this criterion; seven control group animals were included in this study; Fig. $1 B, C$ ), since a pronounced damage to the dorsal sensory and corticospinal tract systems might result in indirect impairment of locomotor function and thereby could potentially bias interpretation of the acquired data.

All spinally injured animals were tested for overground locomotor performance, using a setup described previously (Zörner et al., 2010), which allowed us to assess fine kinematic details of walking, wading, and swimming behavior (Fig. 1D). Locomotor performance was assessed before injury (BL), as well as in the subacute/acute (7 dpi) and chronic ( $84 \mathrm{dpi}$ ) stages following spinal lesioning (Fig. 2D). Kinematic tracking of joint movements allowed us to determine 45 features for each of the locomotor modalities walking and wading (Extended Data Fig. 1-1A), as well as 18 features for swimming (Extended Data Fig. 1-1C), which provided us with detailed insight into the characteristics of baseline performance, as well as the initial impairment and recovery over time. In a first step of analysis, the acquired dataset of single features was categorized based on statistical significance of initial impairment following spinal injury and recovery over time (84 dpi; one-way ANOVA, with Tukey's post hoc test for multiple comparison; $p<0.05$ ). Features that showed statistically significant lesion-induced initial impairment as well as statistically significant spontaneous recovery over 3 months were considered class A parameters. Representative examples of class A features for walking were a base of support of the hindlimbs $\left(p_{\mathrm{BL} / 7 \mathrm{dpi}}=5.55 * 10^{-12} ; p_{7 \mathrm{dpi} / 84 \mathrm{dpi}}=2.18^{*} 10^{-7} ; \mathrm{p}_{\mathrm{BL} / 84 \mathrm{dpi}}=\right.$ $0.027)$, step frequency of the right hindlimb $\left(p_{\mathrm{BL} / 7 \mathrm{dpi}}=1.26 *\right.$ $\left.10^{-12} ; p_{7 \mathrm{dpi} / 84 \mathrm{dpi}}=0.02 ; p_{\mathrm{BL} / 84 \mathrm{dpi}}=2.01 * 10^{-9}\right)$, and protraction of the right hindlimb $\left(p_{\mathrm{BL} / 7 \mathrm{dpi}}=1.61{ }^{*} 10^{-8} ; p_{7 \mathrm{dpi} / 84 \mathrm{dpi}}=0.0029\right.$; $\left.p_{\mathrm{BL} / 84 \mathrm{dpi}}=0.0042\right)$; Figure $1 F$ class A characteristics representative for wading were iliac crest height of the right hindlimb $\left(p_{\mathrm{BL} /}\right.$ $7 \mathrm{dpi}=1.28 * 10^{-12} ; p_{7 \mathrm{dpi} / 84 \mathrm{dpi}}=3.09 * 10^{-6} ; p_{\mathrm{BL} / 84 \mathrm{dpi}}=7.02 *$ 
$\left.10^{-5}\right)$, relative $\mathrm{MCH}$ speed of the right forelimb $\left(p_{\mathrm{BL} / 7 \mathrm{dpi}}=\right.$ $\left.0.0017 ; p_{7 \mathrm{dpi} / 84 \mathrm{dpi}}=0.013 ; p_{\mathrm{BL} / 84 \mathrm{dpi}}=0.89\right)$, and swing phase duration of the right hindlimb $\left(p_{\mathrm{BL} / 7 \mathrm{dpi}}=9.95 * 10^{-12} ; p_{7 \mathrm{dpi} / 84 \mathrm{dpi}}=\right.$ $\left.1.25^{\star} 10^{-5} ; p_{\mathrm{BL} / 84 \mathrm{dpi}}=0.0013\right)$; Figure $1 G$, for swimming, overall speed $\left(p_{\mathrm{BL} / 7 \mathrm{dpi}}=9.38^{\star} 10^{-12} ; p_{7 \mathrm{dpi} / 84 \mathrm{dpi}}=0.033 ; p_{\mathrm{BL} / 84 \mathrm{dpi}}=8.84\right.$ ${ }^{*} 10^{-8}$ ) was identified as the only class A parameter (Fig. $1 H$ ). Features that did not show impairment were classified as class B, and features that did show initial impairment but no significant recovery over time as class $\mathrm{C}$, as for example propulsion phase duration of the right hindlimb during swimming $\left(p_{\mathrm{BL} / 7 \mathrm{dpi}}=1.92\right.$ ${ }^{*} 10^{-7} ; p_{7 \mathrm{dpi} / 84 \mathrm{dpi}}=0.13 ; p_{\mathrm{BL} / 84 \mathrm{dpi}}=2.51{ }^{*} 10^{-4} ;$ Fig. $\left.1 H\right)$. Since the aim of this study was to causally test the functional relevance of different forms of NRG plasticity for functional recovery, further analysis was limited to class A parameters. This filtering step reduced the number of relevant features to 19 for walking, 9 for wading, and 1 for swimming (Fig. $1 E$; class A parameters are highlighted in Figs. 3, 4, 5). Principal component analysis of the filtered datasets for walking and wading showed that the reduced dataset of features was able to robustly discriminate among baseline, acute, and chronic time points (Fig. $1 F, G$ ). Since there was only one feature left following filtering of the swimming dataset, the resulting reduced swimming data did not have the power to reflect lesion-induced behavioral changes over time. We therefore decided to focus our analysis of the causal relevance of different forms of NRG plasticity for recovery of crude motor function on the locomotor modalities walking and wading.

Three months after the injury, when spontaneous recovery has happened and reached a stable level, spinally lesioned animals were assigned to one of two experimental groups. Care was taken to ensure representative and comparable distribution of lesion size and level of recovery in the different groups (Fig. $1 B$, $C, F-H)$. To investigate the relevance of local rewiring and compensatory NRG plasticity for functional recovery, we introduced the inhibitory version of the chemogenetic DREADD receptor hM4Di specifically into either the locally rewired NRG projections (Fig. 2A; "cerv" group, viral injections at sites A and C) or the compensatorily sprouted NRG neurons (Fig. 2A; "lumb" group, viral injections at sites $\mathrm{B}$ and $\mathrm{D}$ ) using projection-specific, intersectional viral genetics (Fig. $2 A-D$ ). Intact animals without hemisection lesions served as control group for potential off-target effects of $\mathrm{CNO}$ treatment or the virus injection procedure and received the full injection scheme (Fig. $2 A, B$; "int" group, viral injections at sites $\mathrm{A}, \mathrm{B}, \mathrm{C}$, and $\mathrm{D})$. This region-specific as well as projection-specific viral targeting approach resulted in efficient and specific labeling of NRG-spinal projection neurons (Fig. 2E,F). On average across all groups, we found 55-99 NRG neurons per $40 \mu \mathrm{m}$ section of the brainstem to be double transfected with both viral vectors and therefore express the hM4Di/ mCherry construct. The number of successfully transfected NRG neurons was comparable between groups, with a clear preference of labeling for the injected ipsilesional side of the NRG in the cervical animals (78 \pm 10 mCherry-positive neurons $/ 40 \mu \mathrm{m}$ section in the ipsilesional vs $9 \pm 1$ in the contralesional NRG), for the injected contralesional NRG in the lumbar group $(99 \pm 11$ mCherry-positive neurons/section in the contralesional NRG vs $10 \pm 3$ in the ipsilesional NRG) and with a bilateral transfection pattern in the intact group (intact: $55 \pm 6$ mCherry-positive neurons/section in the ipsilesional NRG vs $56 \pm 6$ in the contralesional NRG; Fig. 2F). When compared with intact animals, the number of NRG-positive neurons in the lesioned groups was increased, probably reflecting greater local density of NRG fibers because of sprouting at the respective injection sites. To assess potential unspecific chemogenetic targeting, we quantified
mCherry-positive neurons in the $\mathrm{PnC}, \mathrm{MdV}$, and the raphe nuclei, regions within the reticular formation adjacent to the $\mathrm{NRG}$, as well as in the VeN, where they are located along the needle tract (Fig. 2G). Only a small number of labeled neurons was detected in these off-target structures; on average, we detected 3.4 mCherry-positive neurons in these structures, with the highest number detected in the $\mathrm{MdV}$, the region caudal to the NRG (int group: $4 \pm 2$ mCherry-positive neurons/section in the ipsilesional vs $4 \pm 1$ in the contralesional MdV; cerv group: $7 \pm 1$ mCherry-positive neurons/section in the ipsilesional vs $2 \pm 1$ in the contralesional MdV; lumb group: $3 \pm 1$ mCherry-positive neurons/section in the ipsilesional vs $8 \pm 2$ in the contralesional MdV; Fig. 2G).

After a recovery period of 3 weeks following viral injection, animals were behaviorally tested for locomotor performance after application of a vehicle control solution to assess potential behavioral impact because of stereotaxic injections (data not shown), as well as in the presence of the DREADD ligand/neuronal silencer $\mathrm{CNO}$ to investigate functional relevance of NRG-spinal plasticity (Fig. 2D). The optimal dosage and timing of CNO injections to ensure inhibition of the transfected NRG neurons while minimizing potential off-target effects was determined in pilot experiments: stepwise $\mathrm{CNO}$ dose escalation from 1 up to $20 \mathrm{mg} / \mathrm{kg}$ body weight did not result in measurable effects on overground locomotor performance in naive Lewis rats (data not shown). A detailed kinetic study of behavioral effects in spinally lesioned animals with hM4Di receptor expression in the NRG showed robust effects of chemogenetic silencing on locomotion as early as $30 \mathrm{~min}$ following intraperitoneal application of $\mathrm{CNO}$ and lasting for $2 \mathrm{~h}$ (data not shown). Using the projection-specific chemogenetic silencing paradigm in intact rats, we did not detect differences among baseline, vehicle, or $\mathrm{CNO}$ treatment when assessing behavioral performance in walking, wading, or swimming (Figs. $3 G, 4 H, 5 A$, overview), further highlighting the absence of off-target effects of CNO-mediated inhibition or the surgical intervention on NRG output.

For the following overall assessment of lesion-, recovery-, and $\mathrm{CNO}$-induced changes in walking, wading, and swimming, the effect size $(\Delta \mathrm{E})$ was calculated for each feature as a metric to directly visualize these changes in fingerprint plots and heatmaps. Effect size of the SCI-induced recovery of all SCI animals is defined as difference between behavioral performance at 84 versus $7 \mathrm{dpi}$ (pre-CNO effect size), whereas the effect size of $\mathrm{CNO}$-mediated changes is defined as the difference in behavioral performance after the injection of vehicle versus CNO (post$\mathrm{CNO}$ ).

Chemogenetic silencing of NRG neurons in hemisected rats with spinal local rewiring or compensatory fiber growth resulted in robust and widespread reimpairment of stability, speed, and timing aspects of recovered walking function (Fig. $3 A-G$ ). For stability, the base of support of the forelimbs was significantly reimpaired on silencing of newly grown locally rewired or compensatory NRG fibers using CNO $\left(p_{\text {cerv }}=0.035 ; p_{\text {lumb }}=0.006\right.$; Fig. $3 A$ ). For speed, both class A features, the step frequency of both the right hindlimb $\left(p_{\text {cerv }}=0.026 ; p_{\text {lumb }}=0.027\right.$; Fig. $3 D$ ) and left hindlimb $\left(p_{\text {cerv }}=0.018 ; p_{\text {lumb }}=0.018\right.$, Fig. $\left.3 F\right)$, showed robust reimpairment under chemogenetic silencing. For timing, five of the seven class A features showed significant reimpairment of recovered function in the presence of $\mathrm{CNO}$, as follows: swing phase duration of the right forelimb $\left(p_{\text {cerv }}=0.026 ; p_{\text {lumb }}=\right.$ 0.039 ; Fig. $3 E)$; stance phase duration of the left forelimb $\left(p_{\text {cerv }}=\right.$ $\left.0.019 ; p_{\text {lumb }}=0.12\right)$; step cycle duration of both the right hind$\operatorname{limb}\left(p_{\text {cerv }}=0.025 ; p_{\text {lumb }}=0.02\right)$ and left hindlimb $\left(p_{\text {cerv }}=0.021\right.$; 

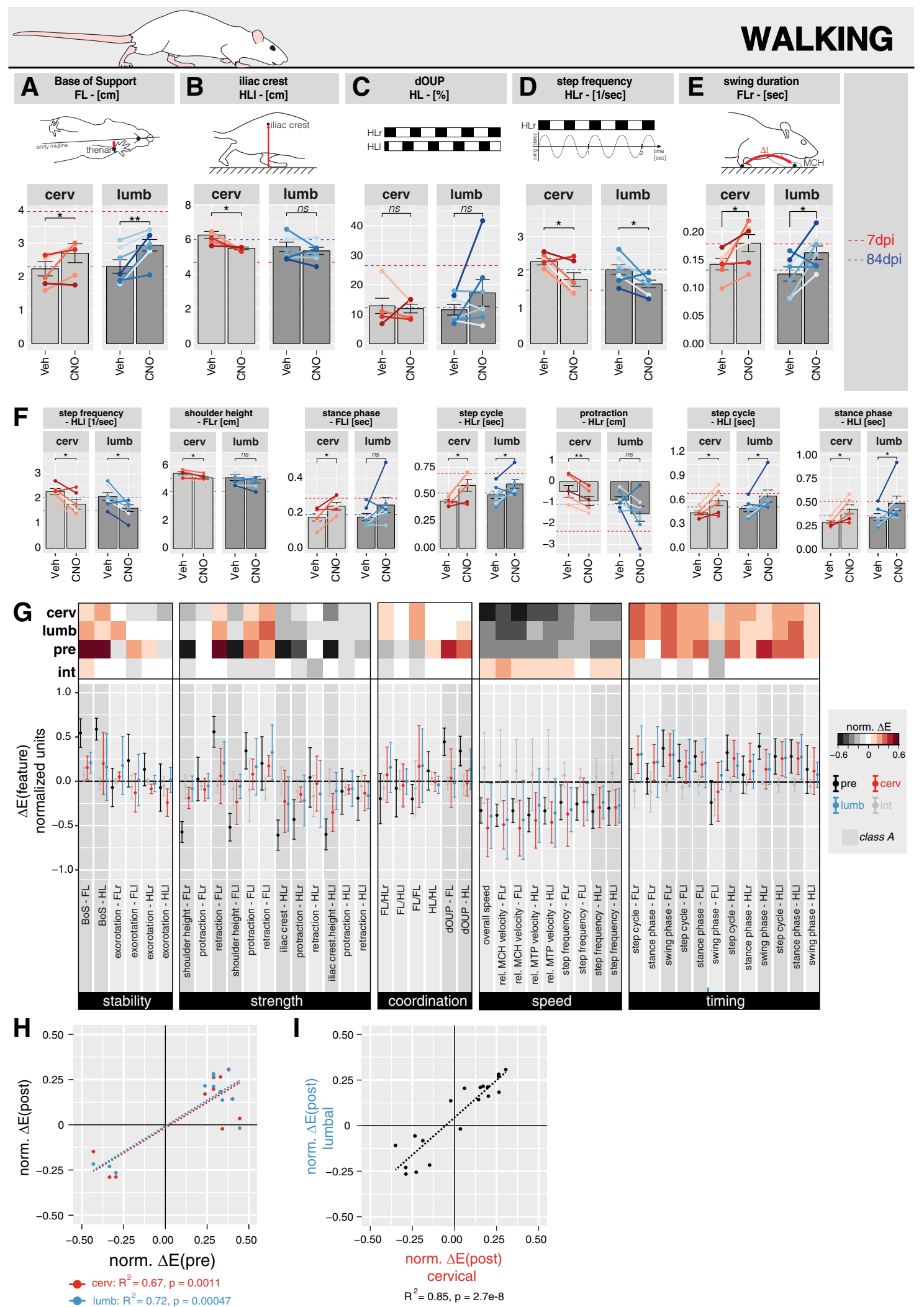

Figure 3. Locally rewired and compensatory NRG plasticity enhance the recovery of stability, speed, and timing of walking in spinal hemisected rats. $\boldsymbol{A}-\boldsymbol{E}$, Representative walking features showing performance across the different groups defined by virus injection (local rewiring of NRG plasticity, cerv/red; compensatory NRG sprouts, lumb/blue) under Veh or CNO treatment (mean \pm SEM, overlaid with single data points for each rat; for color code, see Fig. 1C; statistics: paired $t$ test, $n$ s, not significant, ${ }^{*} p<0.05,{ }^{* *} p<0.001$ ). The five panels show the quantifications of the base of support of the forelimbs $(\boldsymbol{A})$, the height of the iliac crest of the left hindlimb $(\boldsymbol{B})$, the deviation of out of phase of the hindlimbs $(\boldsymbol{C})$, the step frequency of the right hind$\operatorname{limb}(\boldsymbol{D})$, and the swing phase duration of the right forelimb (E). All of those five features have been classified as class $\mathrm{A}$ (significant initial impairment and chronic recovery) at 84 dpi before virus injection (Fig. 1E). In each panel, the dashed red line indicates performance at $7 \mathrm{dpi}$, and the dashed blue line corresponds to performance at 84 dpi. $\boldsymbol{F}$, Remaining class $\mathrm{A}$ walking features showing statistically significant reimpairment of walking function on CNO treatment in any of the different experimental groups (cervical, cerv/red; lumbar, lumb/blue; mean \pm SEM, overlaid 
$\left.p_{\text {lumb }}=0.026\right)$; as well as the swing phase duration of the right $\left(p_{\text {cerv }}=0.015 ; p_{\text {lumb }}=0.039\right)$ and the stance phase duration of the left hindlimb $\left(p_{\text {cerv }}=0.015 ; p_{\text {lumb }}=0.039\right.$; Fig. $\left.3 F\right)$. In contrast, the recovery of strength-related aspects of walking did show a moderate reimpairment phenotype on specific inactivation of locally rewired NRG plasticity (Fig. $3 G$ ). Of the six strengthrelated class A features, shoulder height of the right forelimb $\left(p_{\text {cerv }}=0.012 ; p_{\text {lumb }}=0.22\right.$; Fig. $\left.3 F\right)$, protraction of the right hindlimb $\left(p_{\text {cerv }}=0.008 ; p_{\text {lumb }}=0.124 ;\right.$ Fig. $\left.3 F\right)$, and iliac crest height of the left hindlimb $\left(p_{\text {cerv }}=0.01\right.$; $p_{\text {lumb }}=0.26$; Fig. $3 B$ ) showed significant reimpairment of performance on chemogenetic silencing of locally rewired NRG plasticity. None of the coordination-related class $\mathrm{A}$ features showed significant reimpairment in the presence of $\mathrm{CNO}$ [Fig. 3C; deviation of out of phase (dOUP) of the hindlimbs shown as the representative feature; $p_{\text {cerv }}=0.72, p_{\text {lumb }}=0.21$ ]. In summary, 7 of the 19 class A walking features showed significant reimpairment of performance on chemogenetic silencing of locally rewired or compensatory NRG fiber growth, while 4 of the class A features only showed significant reimpairment in the locally rewired cervical group. Across all class $\mathrm{A}$ features, there is a robust correlation between the pattern of initial impairment and recovery (7 vs $84 \mathrm{dpi}$; pre-CNO experiment) of walking and the detected reimpairment of function on CNO-mediated inactivation of locally rewired $\left(R_{\text {cerv }}^{2}=\right.$ $0.67)$ or compensatory $\left(R_{\text {lumb }}^{2}=0.72\right) \mathrm{NRG}$ plasticity (Veh vs $\mathrm{CNO}$; post-CNO experiment; Fig. $3 H$ ). Additionally, there is a strong correlation between the pattern of CNO treatmentevoked reimpairment between the locally rewired and compensatory datasets $\left(R^{2}=0.85\right.$; Fig. $\left.3 I\right)$.

Chemogenetic silencing of NRG fibers sprouted from intact, contralesional axons (compensatory plasticity) during wading behavior resulted in robust reimpairment of stability, strength,

\section{$\leftarrow$}

with single data points of each group; for color code, see Fig. 1C; statistics: paired $t$ test, $n s$, not significant, ${ }^{*} p<0.05,{ }^{*} p<0.001$ ). In each panel, the dashed red line indicates pre group performance at $7 \mathrm{dpi}$, and the dashed blue line corresponds to pre group performance at 84 dpi. G, Heatmap and fingerprint plot showing the normalized effect size of lesioninduced recovery for all SCl animals (difference between performance at 84 and $7 \mathrm{dpi}$, pre) and CNO dataset for the different experimental groups (difference of performance under vehicle and (NO treatment, int, cerv, and lumb) across all features of walking. The different features are grouped according to distinct aspects of locomotion (stability, strength, coordination, speed, timing), and features that were assigned to class A are highlighted in dark gray. The heatmap visualizes the mean normalized effect size across the different features and groups, based on a color key shown below the figure. The fingerprint plot in the bottom panel shows the normalized effect size across the different features for the pre-virus injection dataset (black) as well as the cervical (red), lumbar (blue), and intact (gray) groups of the CNO dataset (mean $\pm 95 \%$ confidence interval). $\boldsymbol{H}$, Scatterplot of the mean effect size values of the class A features for walking assessed in the cervical (red) and lumbar (blue) groups. On the $x$-axis, the effect size of the pre dataset (difference of performance at 84 and $7 \mathrm{dpi}$ ) is plotted, while the $y$-axis corresponds to the effect size of the post dataset (difference of performance under vehicle and CNO treatment) of the corresponding group. Lines indicate the optimal fit of a linear trendline. For the cervical and the lumbar groups, a clear linear correlation between the two datasets is present (cervical: $R^{2}=0.67, p=0.0011$; lumbar: $R^{2}=$ $0.72, p=0.00,047$; Pearson correlation). $I$, Scatterplot of the mean effect size values of the class $A$ features of the post dataset for walking assessed in the cervical (red) and lumbar (blue) groups. On the $x$-axis, the effect size of the post dataset (difference of performance under vehicle and CNO treatment) for the cervical group is plotted, while the $y$-axis corresponds to the effect size of the post dataset (difference of performance under vehicle and CNO treatment) of the lumbar group. The line indicates the optimal fit of a linear trendline. The cervical and the lumbar post group datasets are strongly correlated $\left(R^{2}=0.85\right.$, $p=2.7 \mathrm{e}-8$; Pearson correlation). pre, Pre group dataset referring to 84 and $7 \mathrm{dpi} ; \Delta \mathrm{E}$, difference between performance at $84 \mathrm{dpi}$ and $7 \mathrm{dpi}$ across all SCl animals in case of predataset, and difference between vehicle and $\mathrm{CNO}$ treatment for post dataset of individual treatment groups; FL, Forelimb; HL, hindlimb; r, right; l, left; BoS, base of support; rel., relative. and speed aspects of recovered function (Fig. 4H). For stability, the base of support of the hindlimbs was significantly reimpaired on silencing of compensatory NRG plasticity using CNO ( $p_{\text {lumb }}$ $=0.03$; Fig. $4 A$ ). For strength, the shoulder height of the left forelimb $\left(p_{\text {lumb }}=0.017\right.$; Fig. $\left.4 E\right)$ as well as the height of the iliac crest of the right hindlimb ( $p_{\text {lumb }}=0.016$; Fig. $4 B$ ) and left hindlimb $\left(p_{\text {lumb }}=0.014\right.$; Fig. $\left.4 E\right)$ were significantly reimpaired. Protraction of the right hindlimb was significantly reimpaired in the locally rewired group ( $p_{\text {cerv }}=0.014$; Fig. $4 E$ ). For speed, the relative $\mathrm{MCH}$ velocity of the right forelimb was significantly reimpaired in the locally rewired group $\left(p_{\text {cerv }}=0.003\right.$; Fig. $4 C$ ), while the compensatory group showed a trend to reimpairment of that feature ( $p_{\text {lumb }}$ $=0.124$ ) on chemogenetic silencing. For timing, no significant changes could be observed in the locally rewired or compensatory condition (Fig. $4 D$, swing phase duration of the right forelimb shown as representative feature; $p_{\text {cerv }}=0.2, p_{\text {lumb }}=0.61$ ).

In summary, four of the nine class A walking features showed significant reimpairment of performance on chemogenetic silencing of compensatory NRG plasticity, while two of the class A features showed significant reimpairment in the locally rewired group. Across all class A features, there is a robust correlation between the pattern of pre-virus injection impairment and the recovery of wading and the detected reimpairment of function on CNO-mediated inactivation of compensatory NRG fibers $\left(R_{\text {lumb }}^{2}=0.71\right)$, while the correlation in the locally rewired group is very limited $\left(R_{\text {cerv }}{ }^{2}=0.15\right.$; Fig. $\left.4 F\right)$. This discrepancy between the effects of chemogenetic silencing of locally rewired versus compensatory NRG plasticity during wading is also shown by the absence of correlation between the two respective datasets $\left(R^{2}=0.074\right.$; Fig. $\left.4 G\right)$.

Initial filtering of the dataset for swimming resulted in only one feature, which was classified as class A (Fig. 1E). CNOinduced silencing showed reimpairment of recovered swimming function, especially under conditions where compensatory NRG sprouting was chemogenetically silenced (Fig. 5A). The one class A feature, overall speed $\left(p_{\text {lumb }}=0.04\right.$; Fig. $\left.5 B\right)$, was significantly reimpaired in the compensatory outgrowth group.

\section{Discussion}

Following unilateral cervical spinal hemisection, human patients (Taylor and Gleave, 1957; Little and Halar, 1985; Roth et al., 1991) and animal models (Webb and Muir, 2002; Rosenzweig et al., 2010; Filli et al., 2011) show pronounced spontaneous recovery of motor function of the lower limbs. Several recent publications have suggested plasticity within the reticular formation, specifically the NRG, to be a neuroanatomical substrate for regained function following incomplete SCI (Ballermann and Fouad, 2006; Courtine et al., 2008; Filli et al., 2014; Zörner et al., 2014; May et al., 2017; Asboth et al., 2018). These studies describe the following different types of reticulospinal plasticity: compensatory outgrowth of spared projections over the midline in the cervical and lumbar cord (Ballermann and Fouad, 2006; Zörner et al., 2014); or local rewiring of axotomized NRG fibers (Courtine et al., 2008; Filli et al., 2014). In the present study, we used the toolbox of chemogenetic neuromodulation to investigate the functional relevance of the two types of bulbospinal plasticity for recovery of walking, wading, and swimming performance in adult, cervically hemisected rats. Intersectional viral genetics allowed differential silencing of locally rewired or compensatory reticulospinal plasticity acutely during behavioral testing. Pronounced reimpairment of stability-, strength-, speed-, and timing-related aspects of forelimbs and hindlimbs during walking, wading, and swimming 

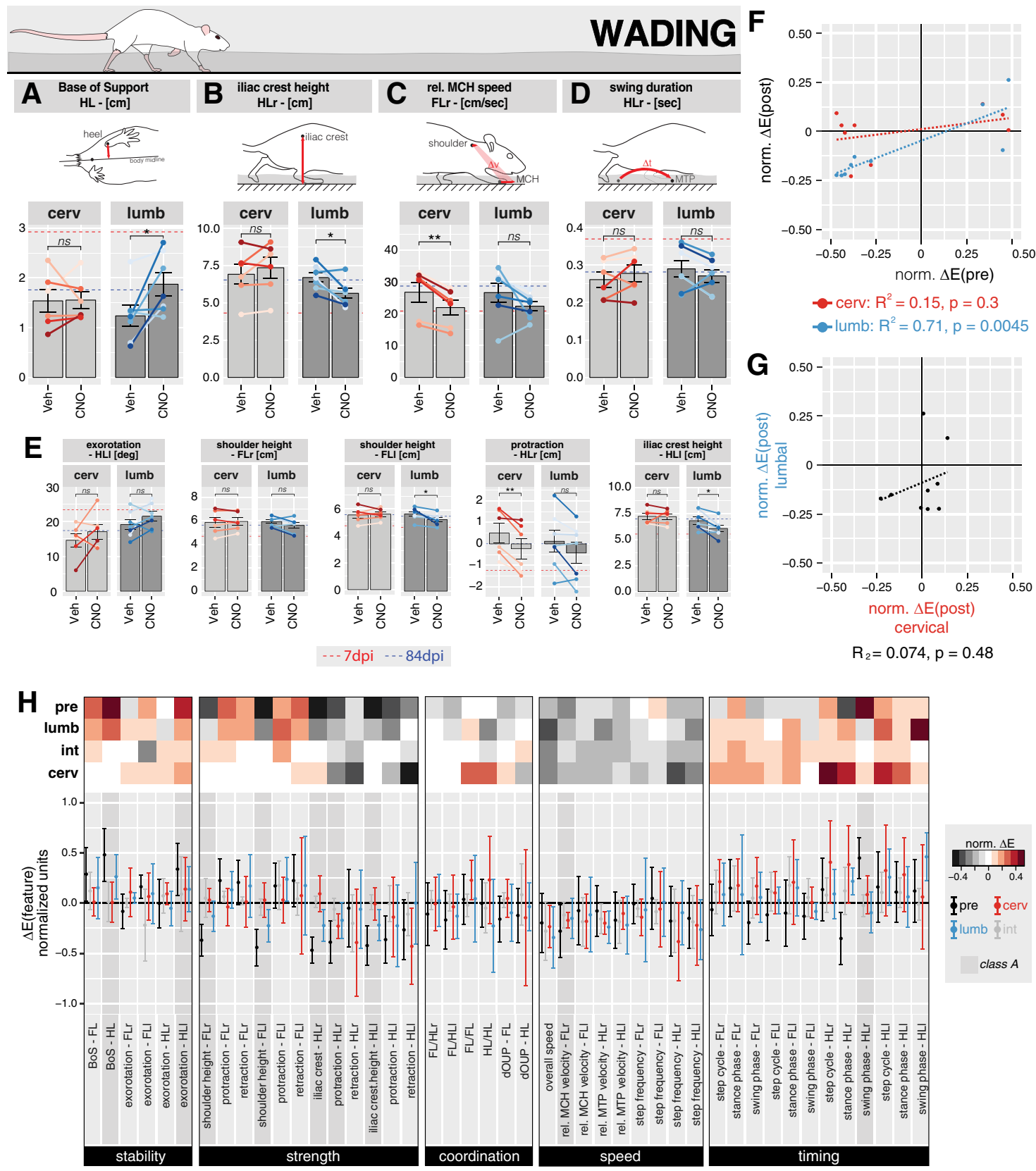

фpre $\oint_{\text {cerv }}$ Ilumb $\Phi$

class $A$

Figure 4. Compensatory and, to lower degrees, local rewiring of NRG plasticity enhance the recovery of stability, speed, and timing of wading in spinal hemisected rats. $\boldsymbol{A}-\boldsymbol{D}$, Representative data for distinct wading features showing performance across the different groups defined by virus injection (locally rewiring NRG sprouts: cerv, red; compensatory NRG sprouts: lumb, blue) under Veh or CNO treatment (mean \pm SEM; overlaid with single data points of each rat; for color code, see Fig. 1C; statistics: paired $t$ test, ns, not significant, ${ }^{*} p<0.05$, ${ }^{* *} p<0.001$ ). The five panels show quantifications of the base of support of the hindlimbs $(\boldsymbol{A})$, the height of the iliac crest of the right hindlimb $(\boldsymbol{B})$, the relative $\mathrm{MCH}$ speed of the right forelimb $(\boldsymbol{C})$, and the swing phase duration of the right hindlimb (D). All of those four features have been classified as class $A$ at 84 dpi before virus injection. In each panel, the dashed red line indicates performance at $7 \mathrm{dpi}$, and the dashed blue line corresponds to performance at 84 dpi. $\boldsymbol{E}$, Representative data for the remaining five class A wading features showing performance across different groups (cerv, red; lumb, blue) under Veh or CNO treatment (mean \pm SEM, overlaid with single data points of each group; Fig. 1C, color code; statistics: paired $t$ test, ns, not significant, ${ }^{*} p<0.05,{ }^{* *} p<0.001$ ). In each panel, the dashed red line indicates pre group performance at $7 \mathrm{dpi}$, and the dashed blue line corresponds to pre group performance at $84 \mathrm{dpi}$. $\boldsymbol{F}$, Scatterplot of the mean effect size values of the class $\mathrm{A}$ features for wading assessed in the cervical (red) and lumbar (blue) groups. On the $x$-axis, the effect size of the pre dataset (difference of performance at 84 and 7 dpi) is plotted, while the $y$-axis corresponds to the effect size of the post dataset (difference of performance under vehicle and CNO treatment) of the corresponding group. Lines indicate the optimal fit of a linear trendline. For the compensatory group, a clear linear correlation between the two datasets is present $\left(R^{2}=0.71, p=0.0045\right.$, Pearson correlation), while this correlation is absent in the case of the cervical group $\left(R^{2}=0.15\right.$, $p=0.3$, Pearson correlation). $G$, Scatterplot of the mean effect size values of the class A features of the post dataset for wading assessed in the cervical (red) and lumbar (blue) groups. On the $x$ axis, the effect size of the post dataset (difference of performance under vehicle and CNO treatment) for the cervical group is plotted, while the $y$-axis corresponds to the effect size of the post dataset (difference of performance under vehicle and CNO treatment) of the lumbar group. The line indicates the optimal fit of a linear trendline. The cervical and the lumbar post group datasets are not correlated $\left(R^{2}=0.074, p=0.48\right.$, Pearson correlation). $\boldsymbol{H}$, Heatmap and fingerprint plot showing the normalized effect size of lesion-induced recovery for all SCl animals (difference between performance at 84 and $7 \mathrm{dpi}$, pre) and CNO dataset for the different experimental groups (difference of performance under vehicle and CNO treatment, int, cerv, and lumb) across all features of wading. The different features are grouped according to distinct aspects of locomotion (stability, strength, speed, timing), and features that were assigned to class A are highlighted in dark gray. The heatmap visualizes the mean normalized effect size across the different features and groups, based on a color key shown below the figure. The fingerprint plot in the bottom panel shows the normalized effect size across the different features for the pre virus injection dataset (black) as well as the cervical (red) and lumbar (blue) groups of the CN0 dataset (mean $\pm 95 \%$ confidence interval). pre, Pre group dataset, here referring to 84 and 7 dpi; FL, Forelimb; HL, hindlimb; r, right; l, left; BoS, base of support; rel., relative. 


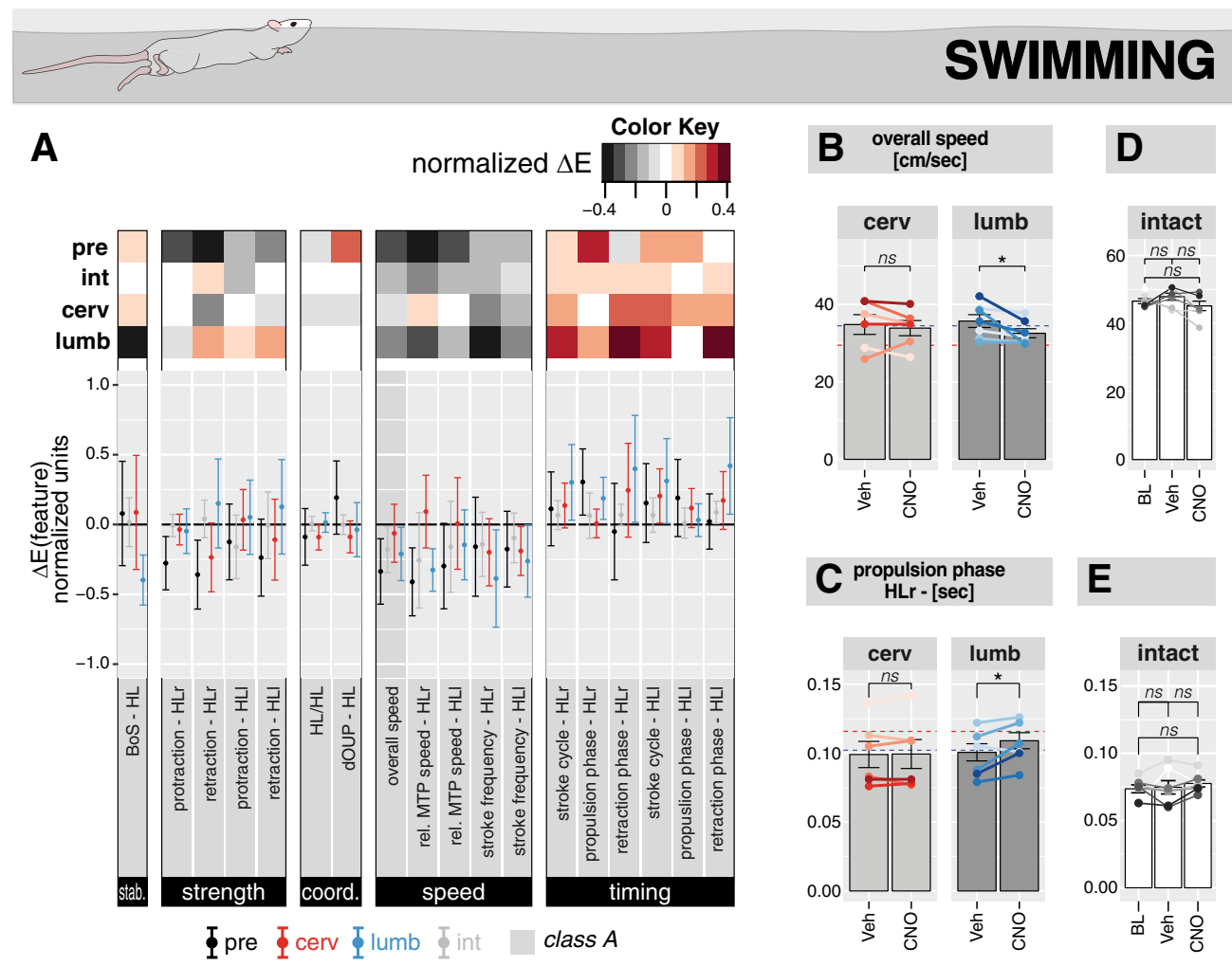

Figure 5. Swimming behavior on chemogenetic silencing of locally rewired or compensatory NRG plasticity. $\boldsymbol{A}$, Heatmap and fingerprint plot showing the normalized effect size of lesioninduced recovery for all SCl animals (difference between performance at 84 and $7 \mathrm{dpi}$, pre) and CNO dataset for the different experimental groups (difference of performance under vehicle and CNO treatment, int, cerv, and lumb) across all 18 features assessed for swimming behavior. The different features are grouped according to distinct aspects of locomotion (stability, strength, coordination, speed, timing). Features that were classified as class A are highlighted in dark gray. The heatmap visualizes the mean normalized effect size across the different features and groups, based on a color key shown in the top right corner. The fingerprint plot in the bottom panel shows the normalized effect size across the different features for the pre virus injection dataset (black), and the cervical (red), compensatory (lumb, blue), and intact (gray) groups of the CNO dataset (mean $\pm 95 \%$ confidence interval). B, C, Representative data for one class A swimming feature, overall speed $(\boldsymbol{B})$, and an example of a class ( feature, propulsion phase duration of the right hindlimb ( $\boldsymbol{C}$, showing performance across the different groups (cerv, red; lumb, blue) under Veh or CNO treatment (mean \pm SEM, overlaid with single data points of each group; Fig. 1C, color code; statistics: paired $t$ test, $n s$, not significant, ${ }^{*} p<0.05$ ). In each panel, the dashed red line indicates pre group performance at $7 \mathrm{dpi}$, and the dashed blue line corresponds to pre group performance at $84 \mathrm{dpi}$. D, E, BL as well as chemogenetic silencing data Veh and CNO treatment for overall speed $(\boldsymbol{D})$ and propulsion phase duration of the right hindlimb $(\boldsymbol{E})$ in the intact control group, showing no significant effect of the surgical intervention or chemogenetic silencing. HL, Hindlimb; r, right; l, left; BoS, base of support; rel., relative.

was observed after the silencing of locally rewired or compensatory circuits. During wading, the observed DREAAD-mediated reimpairment of chronically hemisected animals was more pronounced when compensatory NRG fibers were targeted. These findings demonstrate the causative relevance of reticulospinal plasticity for recovery following hemisection injury. They additionally indicate that different forms of plastic adaptations can be important for differential aspects of functional improvement.

The pattern of initial impairment and subsequent partial recovery of locomotor function following incomplete cervical injury is in line with previously published data (Ballermann and Fouad, 2006; Courtine et al., 2008; Filli et al., 2011, 2014; Zörner et al., 2014; Asboth et al., 2018). While ipsilesional forelimb and hindlimb functions were severely impaired $7 \mathrm{~d}$ after injury, pronounced spontaneous recovery of walking, wading, and swimming performance was present $>4$ weeks after hemisection. This functional recovery has been correlated with spontaneous plasticity of axotomized (Filli et al., 2014) and spared reticulospinal projections (Ballermann and Fouad, 2006; Zörner et al., 2014), which are evolutionarily highly conserved (Butler and Hodos, 2005) and present in lower vertebrate species known to recover from SCI (Cabelguen et al., 2013; Rasmussen and Sagasti, 2017). We used detailed kinematic analyses to assess locomotor deficits and recovery after cervical hemisection. While only a few of the kinematic features did not show impairment after injury, about half of the features showed impairment without recovery, and 19 of 45 walking features, 9 of 45 wading features, as well as 1 of 18 swimming features showed nearly full recovery of initially impaired function. The latter were grouped as class A features. The limited number of class A features for walking and wading reflects the incompleteness of locomotor recovery and the fact that compensatory movement strategies are used during recovery. For swimming, a high proportion of kinematic features (8 of 18) did not show initial impairment, and only one feature did show initial impairment followed by significant recovery. This reflects the defined localization of the execution of swimming behavior to intraspinal circuits of the lumbar cord in limbed animals (Kjaerulff and Kiehn, 1996; Kremer and Lev-Tov, 1997). During swimming, supraspinal control is mostly needed for the initiation of movement (Goulding, 2009; Kiehn, 2011), and following incomplete SCI sufficient descending projections are spared to preserve swimming behavior.

Intersectional viral targeting was applied to efficiently and specifically prime NRG-spinal neurons for chemogenetic silencing: a combination of focused local NRG injections of AAV:: DIO-hM4Di-mCherry and strictly unilateral spinal injections of AAV2.6::Cre enabled region-specific as well as projection-specific targeting of NRG-spinal fibers, with very limited off-target labeling of other tract systems. AAV2.6 had been highlighted before in other tract systems and species as a very efficient 
retrograde viral vector (Löw et al., 2013; Salegio et al., 2013; San Sebastian et al., 2013), an observation that we confirmed for NRG-spinal projections in the rat using AAV2.6::GFP (data not shown). Within the NRG, the majority of doublelabeled, and hence mCherry-positive, neurons were ipsilateral to the lesion and spinal injection site for the locally rewired (cervical) group and contralateral to lesion and spinal injection site for the compensatory (lumbar) group, which is in line with expectations because of the unilaterality of the AAV::DIO-hM4Di-mCherry injections in these groups. Approximately $10 \%$ of labeled neurons were found on the respective contralateral sides, which can be accounted for by retrograde uptake of the Cre-dependent DREADD virus because of the strongly interconnected sides of the NRG as well as its previously reported bilateral spinal projection pattern (Nathan et al., 1996; Bachmann et al., 2014; Takeoka et al., 2014; Zörner et al., 2014; Liang et al., 2016). In the cervical spinal cord, the viral injections target sprouted axotomized NRG-spinal fiber as well as NRG projections, which would naturally terminate in the respective injected spinal segments. Because of a lack of specific histologic or genetic markers for NRG-spinal neurons, quantitative assessment of overall efficacy of chemogenetic priming is not feasible. However, comparison with previously published datasets indicates successful and efficient NRG-spinal targeting: Zörner et al. (2014) and Filli et al. (2014) detected on average 15-17 retrogradely labeled neurons per side and a $40 \mu \mathrm{m} \mathrm{sec}-$ tion of the NRG in intact animals, using very focal, spinal injections of fluorescent dextran tracers. Our intersectional viral injection strategy in intact rats yielded on average 55 labeled NRG-spinal neurons per $40 \mu \mathrm{m}$ section, indicating successful optimization of the injection approach.

The behavioral performance of chemogenetically primed SCI animals treated with vehicle solution was highly comparable to recovered performance before viral injections ( 2 of 108 assessed behavioral features showed significant differences between $84 \mathrm{dpi}$ and Veh; data not shown), indicating that the surgical intervention of viral injections as well as potential associated plastic changes did not significantly affect behavioral readouts.

Acute chemogenetic silencing of NRG-spinal neurons in chronically recovered, behaving SCI animals resulted in reimpairment of various kinematic parameters, as follows: 11 of 19 for walking, 6 of 9 for wading, and 1 of 1 originally recovered features for swimming. The reimpairment was transitory and disappeared with the washout of the channelactivating drug CNO. These results demonstrate a direct functional relevance of respective reticulospinal projections for the recovery of motor performance. They are in line with previous observations using permanent electrolytic lesions of the NRG or manipulations of the cortico-reticular-spinal pathway (Zörner et al., 2014; Asboth et al., 2018). The observation that NRG-spinal silencing in intact rats did not affect walking, wading, or swimming performance also is in line with earlier findings (Zörner et al., 2014). In addition to highlighting the absence of off-target effects with the chosen $\mathrm{CNO}$-mediated inactivation paradigm, this result reflects the functional redundancy of reticulospinal projections in the intact animal (Lemon, 2008; Angeles Fernández-Gil et al., 2010).

Of particular interest was the assessment of the relative functional contribution of locally rewired plasticity of transected NRG axons versus compensatory outgrowth of spared
NRG axons across the midline of the lumbar spinal cord. In the more complex walking task, silencing of either locally rewired or compensatory NRG projections caused significant reimpairment of the stability of the forelimbs, the strength of the ipsilesional hindlimb, and the overall speed- and timingrelated aspects of the movement, while no changes were detected on features related to coordination. These results show that both types of NRG rewiring contribute to the recovery of these important locomotor functions. In contrast to walking, the recovery of wading was more selectively dependent on compensatory NRG sprouting: chemogenetic silencing of spared NRG fibers innervating deafferented lumbar areas by midline crossing robustly reimpaired stability and strength of wading movements, while the inhibition of ipsilesional, locally rewired NRG fibers only had minor effects. Behavioral effects of chemogenetic silencing of locally rewired or compensatory NRG plasticity did not show a distinct forelimb- or hindlimb-specific pattern. This is a direct reflection of the coordinated and interwoven plastic response of bulbospinal projections across different segments and areas of brainstem and spinal cord, tightly coupling recovery of forelimbs and hindlimbs. The difference but also the coexistence and possible synergy of the locally rewiring and compensatory plastic pathways are interesting as both necessitate larger-scale adaptations of the circuitry, as follows: NRG neurons, which project new midline-crossing fibers to the contralateral hemicord, have to adjust their upstream input to enable a functional side-switch. Axotomized NRG neurons, which locally rewire and form detour pathways via propriospinal neurons, have to adjust the timing of motor commands to account for the presence of additional synaptic contacts. The simultaneous occurrence of these different forms of plasticity has also been observed for corticospinal projections in the presence of various types of growth-enhancing treatments (Thallmair et al., 1998; Raineteau et al., 1999; Bareyre et al., 2004; Barritt et al., 2006) and therefore can be seen as a general phenomenon of the plasticity of descending tracts.

\section{References}

Angeles Fernández-Gil M, Palacios-Bote R, Leo-Barahona M, Mora-Encinas JP (2010) Anatomy of the brainstem: a gaze into the stem of life. Semin Ultrasound CT MR 31:196-219.

Asboth L, Friedli L, Beauparlant J, Martinez-Gonzalez C, Anil S, Rey E, Baud L, Pidpruzhnykova G, Anderson MA, Shkorbatova P, Batti L, Pagès S, Kreider J, Schneider BL, Barraud Q, Courtine G (2018) Cortico-reticulospinal circuit reorganization enables functional recovery after severe spinal cord contusion. Nat Neurosci 21:576-588.

Bachmann LC, Matis A, Lindau NT, Felder P, Gullo M, Schwab ME (2013) Deep brain stimulation of the midbrain locomotor region improves paretic hindlimb function after spinal cord injury in rats. Sci Transl Med 5:208ra146.

Bachmann LC, Lindau NT, Felder P, Schwab ME (2014) Sprouting of brainstem-spinal tracts in response to unilateral motor cortex stroke in mice. J Neurosci 34:3378-3389.

Baker SN, Perez MA (2017) Reticulospinal contributions to gross hand function after human spinal cord injury. J Neurosci 37:9778-9784.

Ballermann M, Fouad K (2006) Spontaneous locomotor recovery in spinal cord injured rats is accompanied by anatomical plasticity of reticulospinal fibers. Eur J Neurosci 23:1988-1996.

Bareyre FM, Kerschensteiner M, Raineteau O, Mettenleiter TC, Weinmann O, Schwab ME (2004) The injured spinal cord spontaneously forms a new intraspinal circuit in adult rats. Nat Neurosci 7:269-277.

Barritt AW, Davies M, Marchand F, Hartley R, Grist J, Yip P, McMahon SB, Bradbury EJ (2006) Chondroitinase ABC promotes sprouting of intact and injured spinal systems after spinal cord injury. J Neurosci 26:1085610867. 
Brownstone RM, Chopek JW (2018) Reticulospinal systems for tuning motor commands. Front Neural Circuits 12:30.

Butler AB, Hodos W (2005) Comparative vertebrate neuroanatomy: evolution and adaptation. Ed 2. Hoboken, NJ: Wiley-Interscience.

Cabelguen JM, Chevallier S, Amontieva-Potapova I, Philippe C (2013) Anatomical and electrophysiological plasticity of locomotor networks following spinal transection in the salamander. Neurosci Bull 29:467-476.

Caggiano V, Leiras R, Goñi-Erro H, Masini D, Bellardita C, Bouvier J, Caldeira V, Fisone G, Kiehn O (2018) Midbrain circuits that set locomotor speed and gait selection. Nature 553:455-460.

Courtine G, Song B, Roy RR, Zhong H, Herrmann JE, Ao Y, Qi J, Edgerton VR, Sofroniew MV (2008) Recovery of supraspinal control of stepping via indirect propriospinal relay connections after spinal cord injury. Nat Med 14:69-74.

Dobkin B, Barbeau H, Deforge D, Ditunno J, Elashoff R, Apple D, Basso M, Behrman A, Fugate L, Harkema S, Saulino M, Scott M (2007) The evolution of walking-related outcomes over the first 12 weeks of rehabilitation for incomplete traumatic spinal cord injury: the multicenter randomized Spinal Cord Injury Locomotor Trial. Neurorehabil Neural Repair 21:25-35.

Fawcett JW, Curt A, Steeves JD, Coleman WP, Tuszynski MH, Lammertse D, Bartlett PF, Blight AR, Dietz V, Ditunno J, Dobkin BH, Havton LA, Ellaway PH, Fehlings MG, Privat A, Grossman R, Guest JD, Kleitman N, Nakamura M, Gaviria M, et al (2007) Guidelines for the conduct of clinical trials for spinal cord injury as developed by the ICCP panel: spontaneous recovery after spinal cord injury and statistical power needed for therapeutic clinical trials. Spinal Cord 45:190-205.

Fenno L, Yizhar O, Deisseroth K (2011) The development and application of optogenetics. Annu Rev Neurosci 34:389-412.

Filli L, Zörner B, Weinmann O, Schwab ME (2011) Motor deficits and recovery in rats with unilateral spinal cord hemisection mimic the BrownSequard syndrome. Brain 134:2261-2273.

Filli L, Engmann AK, Zörner B, Weinmann O, Moraitis T, Gullo M, Kasper H, Schneider R, Schwab ME (2014) Bridging the gap: a reticulo-propriospinal detour bypassing an incomplete spinal cord injury. J Neurosci 34:13399-13410.

Fouad K, Tse A (2008) Adaptive changes in the injured spinal cord and their role in promoting functional recovery. Neurol Res 30:17-27.

Gompf HS, Budygin EA, Fuller PM, Bass CE (2015) Targeted genetic manipulations of neuronal subtypes using promoter-specific combinatorial AAVs in wild-type animals. Front Behav Neurosci 9:152.

Goulding M (2009) Circuits controlling vertebrate locomotion: moving in a new direction. Nat Rev Neurosci 10:507-518.

Hasan SJ, Keirstead HS, Muir GD, Steeves JD (1993) Axonal regeneration contributes to repair of injured brainstem-spinal neurons in embryonic chick. J Neurosci 13:492-507.

Jordan LM, Liu J, Hedlund PB, Akay T, Pearson KG (2008) Descending command systems for the initiation of locomotion in mammals. Brain Res Rev 57:183-191.

Kiehn O (2011) Development and functional organization of spinal locomotor circuits. Curr Opin Neurobiol 21:100-109.

Kjaerulff O, Kiehn O (1996) Distribution of networks generating and coordinating locomotor activity in the neonatal rat spinal cord in vitro: a lesion study. J Neurosci 16:5777-5794.

Kremer E, Lev-Tov A (1997) Localization of the spinal network associated with generation of hindlimb locomotion in the neonatal rat and organization of its transverse coupling system. J Neurophysiol 77:1155-1170.

Lemon RN (2008) Descending pathways in motor control. Annu Rev Neurosci 31:195-218.

Liang H, Watson C, Paxinos G (2016) Terminations of reticulospinal fibers originating from the gigantocellular reticular formation in the mouse spinal cord. Brain Struct Funct 221:1623-1633.

Little JW, Halar E (1985) Temporal course of motor recovery after BrownSequard spinal cord injuries. Paraplegia 23:39-46.

Löw K, Aebischer P, Schneider BL (2013) Direct and retrograde transduction of nigral neurons with AAV6, 8, and 9 and intraneuronal persistence of viral particles. Hum Gene Ther 24:613-629.

Lurie DI, Selzer ME (1991) Axonal regeneration in the adult lamprey spinal cord. J Comp Neurol 306:409-416.
May Z, Fenrich KK, Dahlby J, Batty NJ, Torres-Espín A, Fouad K (2017) Following spinal cord injury transected reticulospinal tract axons develop new collateral inputs to spinal interneurons in parallel with locomotor recovery. Neural Plast 2017:1932875.

Mori S, Matsui T, Kuze B, Asanome M, Nakajima K, Matsuyama K (1998) Cerebellar-induced locomotion: reticulospinal control of spinal rhythm generating mechanism in cats. Ann N Y Acad Sci 860:94-105.

Müllner A, Gonzenbach RR, Weinmann O, Schnell L, Liebscher T, Schwab ME (2008) Lamina-specific restoration of serotonergic projections after Nogo-A antibody treatment of spinal cord injury in rats. Eur J Neurosci 27:326-333.

Nathan PW, Smith M, Deacon P (1996) Vestibulospinal, reticulospinal and descending propriospinal nerve fibres in man. Brain 119:18091833.

Noga BR, Kettler J, Jordan LM (1988) Locomotion produced in mesencephalic cats by injections of putative transmitter substances and antagonists into the medial reticular formation and the pontomedullary locomotor strip. J Neurosci 8:2074-2086.

Noga BR, Kriellaars DJ, Brownstone RM, Jordan LM (2003) Mechanism for activation of locomotor centers in the spinal cord by stimulation of the mesencephalic locomotor region. J Neurophysiol 90:1464-1478.

Orlovskii GN (1970) Work of reticulo-spinal neurons during locomotion (in Russian). Biofizika 15:728-737.

Paxinos G, Watson C (1998) The rat brain in stereotaxic coordinates. Ed 4. San Diego: Academic.

Pettersson LG, Alstermark B, Blagovechtchenski E, Isa T, Sasaski S (2007) Skilled digit movements in feline and primate-recovery after selective spinal cord lesions. Acta Physiol (Oxf) 189:141-154.

Raineteau O, Schwab ME (2001) Plasticity of motor systems after incomplete spinal cord injury. Nat Rev Neurosci 2:263-273.

Raineteau O, Z'Graggen WJ, Thallmair M, Schwab ME (1999) Sprouting and regeneration after pyramidotomy and blockade of the myelin-associated neurite growth inhibitors NI 35/250 in adult rats. Eur J Neurosci 11:1486-1490.

Rasmussen JP, Sagasti A (2017) Learning to swim, again: axon regeneration in fish. Exp Neurol 287:318-330.

Roseberry TK, Lee AM, Lalive AL, Wilbrecht L, Bonci A, Kreitzer AC (2016) Cell-type-specific control of brainstem locomotor circuits by basal ganglia. Cell 164:526-537.

Rosenzweig ES, Courtine G, Jindrich DL, Brock JH, Ferguson AR, Strand SC, Nout YS, Roy RR, Miller DM, Beattie MS, Havton LA, Bresnahan JC, Edgerton VR, Tuszynski MH (2010) Extensive spontaneous plasticity of corticospinal projections after primate spinal cord injury. Nat Neurosci 13:1505-1510.

Roth BL (2016) DREADDs for neuroscientists. Neuron 89:683-694.

Roth EJ, Park T, Pang T, Yarkony GM, Lee MY (1991) Traumatic cervical Brown-Sequard and Brown-Sequard-plus syndromes: the spectrum of presentations and outcomes. Paraplegia 29:582-589.

Salegio EA, Samaranch L, Kells AP, Mittermeyer G, San Sebastian W, Zhou S, Beyer J, Forsayeth J, Bankiewicz KS (2013) Axonal transport of adeno-associated viral vectors is serotype-dependent. Gene Ther 20:348-352.

San Sebastian W, Samaranch L, Heller G, Kells AP, Bringas J, Pivirotto P, Forsayeth J, Bankiewicz KS (2013) Adeno-associated virus type 6 is retrogradely transported in the non-human primate brain. Gene Ther 20:1178-1183.

Shik ML, Severin FV, Orlovskiü GN (1966) [Control of walking and running by means of electric stimulation of the midbrain]. Biofizika 11:659-666 (in Russian).

Sinnamon HM, Stopford CK (1987) Locomotion elicited by lateral hypothalamic stimulation in the anesthetized rat does not require the dorsal midbrain. Brain Res 402:78-86.

Skinner RD, Garcia-Rill E (1984) The mesencephalic locomotor region (MLR) in the rat. Brain Res 323:385-389.

Steeves JD, Sholomenko GN, Webster DM (1987) Stimulation of the pontomedullary reticular formation initiates locomotion in decerebrate birds. Brain Res 401:205-212.

Takeoka A, Vollenweider I, Courtine G, Arber S (2014) Muscle spindle feedback directs locomotor recovery and circuit reorganization after spinal cord injury. Cell 159:1626-1639. 
Taylor RG, Gleave JR (1957) Incomplete spinal cord injuries; with BrownSequard phenomena. J Bone Joint Surg Br 39-B(3):438-450.

Thallmair M, Metz GA, Z'Graggen WJ, Raineteau O, Kartje GL, Schwab ME (1998) Neurite growth inhibitors restrict plasticity and functional recovery following corticospinal tract lesions. Nat Neurosci $1: 124-131$.

Vavrek R, Pearse DD, Fouad K (2007) Neuronal populations capable of regeneration following a combined treatment in rats with spinal cord transection. J Neurotrauma 24:1667-1673.

Webb AA, Muir GD (2002) Compensatory locomotor adjustments of rats with cervical or thoracic spinal cord hemisections. J Neurotrauma 19:239-256.
Zaaimi B, Edgley SA, Soteropoulos DS, Baker SN (2012) Changes in descending motor pathway connectivity after corticospinal tract lesion in macaque monkey. Brain 135:2277-2289.

Zörner B, Filli L, Starkey ML, Gonzenbach R, Kasper H, Röthlisberger M, Bolliger M, Schwab ME (2010) Profiling locomotor recovery: comprehensive quantification of impairments after CNS damage in rodents. Nat Methods 7:701-708

Zörner B, Bachmann LC, Filli L, Kapitza S, Gullo M, Bolliger M, Starkey ML, Röthlisberger M, Gonzenbach RR, Schwab ME (2014) Chasing central nervous system plasticity: the brainstem's contribution to locomotor recovery in rats with spinal cord injury. Brain 137:17161732 . 